\title{
ESTIMATION OF SPECTRAL GAP FOR ELLIPTIC OPERATORS
}

\author{
MU-FA CHEN AND FENG-YU WANG
}

\begin{abstract}
A variational formula for the lower bound of the spectral gap of an elliptic operator is presented in the paper for the first time. The main known results are either recovered or improved. A large number of new examples with sharp estimate are illustrated. Moreover, as an application of the march coupling, the Poincaré inequality with respect to the absolute distribution of the process is also studied.
\end{abstract}

\section{INTRODUCTION}

Consider the operator $L=\sum_{i, j=1}^{d} a_{i j}(x) \partial_{i} \partial_{j}+\sum_{i=1}^{d} b_{i}(x) \partial_{i}$, where $\partial_{i}=\frac{\partial}{\partial x_{i}}$, $a(x):=\left(a_{i j}(x)\right)$ is positive definite, $a_{i j} \in C^{2}\left(\mathbf{R}^{d}\right)$ and $b_{i}=\sum_{j=1}^{d}\left(a_{i j} \partial_{j} V+\partial_{j} a_{i j}\right)$ for some $V \in C^{2}\left(\mathbf{R}^{d}\right)$ with $Z:=\int \exp [V(x)] \mathrm{d} x<\infty$. We denote $L$ by $L \sim(a, b)$ or $L \sim(a, V)$ and let $\pi(\mathrm{d} x)=Z^{-1} \exp [V(x)] \mathrm{d} x$.

Throughout of this paper, we assume that the $L$-diffusion process is non-explosive so that the corresponding Dirichlet form is regular. Then the first (non-trivial) eigenvalue $\lambda_{1}$ or the spectral gap can be characterized as

$$
\operatorname{gap}(L)=\inf \left\{\pi(\langle a \nabla f, \nabla f\rangle): f \in \mathcal{D}, \pi(f)=0, \pi\left(f^{2}\right)=1\right\},
$$

where $\pi(f)=\int f \mathrm{~d} \pi$ and $\mathcal{D}=\left\{f+c: f \in C_{0}^{\infty}\left(\mathbf{R}^{d}\right), c \in \mathbf{R}\right\}$. The variational formula (1.1) is particularly useful for a upper bound of $\operatorname{gap}(L)$. But it is much more difficult to handle the lower bound for which many different approaches have been introduced. The readers are urged to refer to [6] for further comments and references.

To show the difficulty of the problem, we mention here three simple examples. Let $d=1$ and take $a \equiv 1, b(x)=-x$. Then the first eigenvalue is $\lambda_{1}=1$. We now go to the half line $[0, \infty)$ with reflecting boundary and with the same $a$. Then $\lambda_{1}=2$ or 3 according as $b(x)=-x$ or $-(x+1)$. Surprisingly, the order of the corresponding eigenfunctions changes from 1, 2 to 3 successively. From these, one sees that the first eigenvalue is very sensitive.

To get some impression about the results obtained in the paper, let us restrict ourselves to the half line $[0, \infty)$. Denote by $\mathcal{F}$ the set of all functions $f \in L^{1}(\pi)$ with $f^{\prime}>0$ on $(0, \infty)$. Define $C(x)=\int_{0}^{x} a(y)^{-1} b(y) \mathrm{d} y$. We will use quite often the

Received by the editors December 3, 1995

1991 Mathematics Subject Classification. Primary 35P15, $60 \mathrm{H} 30$.

Key words and phrases. Spectral gap, diffusion process, coupling.

Research supported in part by the National Natural Science Foundation of China and the Foundation of Institution of Higher Education for Doctoral Program.

(C)1997 American Mathematical Society 
following mapping $I: \mathcal{F} \rightarrow C[0, \infty)$ or its variations.

$$
I(f)(x)=\frac{e^{-C(x)}}{f^{\prime}(x)} \int_{x}^{\infty} \frac{f(u) e^{C(u)}}{a(u)} \mathrm{d} u=\frac{e^{-V(x)}}{a(x) f^{\prime}(x)} \int_{x}^{\infty} f(u) e^{V(u)} \mathrm{d} u, \quad x>0, f \in \mathcal{F} .
$$

Then, we have

$$
\operatorname{gap}_{[0, \infty)} \geq \sup _{f \in \mathcal{F}} \inf _{x>0} I(f)(x)^{-1}
$$

This is an alternative statement of Theorem 2.1 (2) given below. No doubt, this is a very convenient formula since it is usually quite easy to choose a test function $f \in \mathcal{F}$ to obtain a non-trivial estimate. Moreover, it is proved that equality in (1.3) actually holds in the regular case (cf. Proposition 6.4). This new variational formula is clearly a dual of (1.1). It is remarkable that the two formulas have no common point.

This paper is based on a new probabilistic method, i.e. the coupling approach, introduced by the authors in [5] and further developed in [3], [6], [15] and [16]. For the reader's convenience, let us explain briefly the main ideas of the method. First, we construct some degenerated elliptic operators $\widetilde{L}$ on the product space $\mathbf{R}^{d} \times \mathbf{R}^{d}$ so that $\widetilde{L} f_{i}\left(x_{1}, x_{2}\right)=L f\left(x_{i}\right)$ for $i=1,2$, all $f \in C_{b}^{2}\left(\mathbf{R}^{d}\right)$ and all $x_{1} \neq x_{2}$, where $f_{i}\left(x_{1}, x_{2}\right)=f\left(x_{i}\right), i=1,2, x_{1}, x_{2} \in \mathbf{R}^{d}$. The operator $\widetilde{L}$ is then called a coupling of $L$ (see [3] or [4] for details). Next, choose a distance $d(x, y)$ in $\mathbf{R}^{d}$. Our main estimate comes from the following inequality

$$
\widetilde{L} d(x, y) \leq-\delta d(x, y), \quad \text { for all } x \neq y
$$

where $\widetilde{L}$ is a coupling operator and $\delta>0$ is a constant. From this, we deduce that $\operatorname{gap}(L) \geq \delta$. Certainly, we have ignored a lot of technical points in this step. Anyhow, from (1.4), one sees that the estimate depends heavily on the choice of both the coupling operator $\widetilde{L}$ and the distance $d(x, y)$. On the other hand, it is known from [3] that the couplings $\widetilde{L}$ can be classified according to different classes of distances and moreover for each class (usually quite large) of distances, there often (sometimes uniquely) exists an optimal $\widetilde{L}$. Therefore, constructing a "good" distance plays a critical role in the study of estimates of the spectral gap (as well as many applications of the coupling approach), as illustrated in our recent publications.

The second key point of our method is that the eigenfunction of $\lambda_{1}$ has to be Lipschitz with respect to the distance adopted. This once again gives the choice of the distance a serious influence on the effectiveness of the approach, especially for non-compact spaces. From this point of view, our approach seems quite restrictive. For instance, in [6] we were unable to cover completely the one-dimensional case for which we employed an analytic approach, a continuous analog of [13]. However, this serious problem turns out to be helpful. It provides us a way to construct some effective distances. That is, roughly speaking, choosing the distance from the eigenfunction or its approximations. Fortunately, this idea is successful as one will see soon in the next section. This paper should be considered as a critical step in the study of couplings and the idea of the paper should be useful in various applications of the coupling method as well as in the study of related topics.

Since the topic is quite technical as one can imagine, we choose a special way to organize the paper. Starting from the simplest case, i.e. the half line (Section 2), 
then go to the full line (Section 3) and finally studying the general case (Section 4). In each section, we introduce the results, explain the ideas and present a large number of examples (which should be considered as a critical part of the paper) in illustration of the results. One sees in a gradual way how the ideas move from a special case to the general one. The proofs are shorter than the statements of the results. Having some preparations (Section 5) at hand, the proofs of the results (except one) of Sections $2-4$ are given in Sections $6-8$ respectively. The equality in (1.3) is explored at the end of Section 6 and Section 7.

This paper is a continuation of [6] but it is nevertheless self-contained. Some ideas come from our previous papers, not only from the study on the estimate of the spectral gap but also from the study of the estimate of Logarithmic Sobolev inequality (see [7], [16], [17] and references therein). Besides, the paper is also an interaction with the study of the same topic for Markov chains and with the study on path space $([10],[17])$. In particular, a result on the Poincaré inequality with respect to the absolute distributions of the process is included in Section 4 and proved in Section 9. Finally, the paper [12], introduced to one of the authors by S. Kotani, is very helpful.

\section{The Case of the Half Line}

Consider a reflecting diffusion on the half line $\left[x_{0}, \infty\right)$ with operator $L \sim(a, b)$. Set $C(x)=\int_{x_{0}}^{x} b(u) a(u)^{-1} \mathrm{~d} u$. Then, the condition " $Z<\infty$ " and the well-known Feller non-explosive criterion can be stated as follows.

$$
Z=\int_{x_{0}}^{\infty} \frac{e^{C(x)}}{a(x)} \mathrm{d} x<\infty, \quad \int_{x_{0}}^{\infty} \mathrm{d} x e^{-C(x)} \int_{x_{0}}^{x} \frac{e^{C(y)}}{a(y)} \mathrm{d} y=\infty .
$$

The left-end point of the half line is not essential in this section but it will be critical in the next section. To emphasize the half line, we use $\operatorname{gap}_{\left[x_{0}, \infty\right]}$ instead of $\operatorname{gap}(L)$. Recall that the mapping $I(f)$ was defined in (1.2) but in which the function $C(x)$ is replaced by the one just defined here.

Theorem 2.1. Assume that (2.1) holds.

(1) For every function $f \in C^{1}\left[x_{0}, \infty\right) \cap C^{2}\left(x_{0}, \infty\right)$ with $f>0$ on $\left(x_{0}, \infty\right)$, we have

$$
\begin{aligned}
\operatorname{gap}_{\left[x_{0}, \infty\right]} & \geq \inf _{x>x_{0}}\left[\left(-a f^{\prime}-b f\right)^{\prime} / f\right](x) \\
& =\inf _{x>x_{0}}\left[-b^{\prime}-\left(a f^{\prime \prime}+\left(a^{\prime}+b\right) f^{\prime}\right) / f\right](x) .
\end{aligned}
$$

(2) For every function $f \in C\left[x_{0}, \infty\right) \cap C^{1}\left(x_{0}, \infty\right) \cap L^{1}(\pi)$ with $\pi(f) \geq 0$ and $f^{\prime}>0$ on $\left(x_{0}, \infty\right)$, we have

$$
\operatorname{gap}_{\left[x_{0}, \infty\right)} \geq \inf _{x>x_{0}} I(f)(x)^{-1} .
$$

In particular, if moreover $f \in C^{2}\left[x_{0}, \infty\right)$, then

$$
\operatorname{gap}_{\left[x_{0}, \infty\right]} \geq c \text { provided }-\left(a f^{\prime \prime}+b f^{\prime}\right) \geq c f \text { for some } c>0 .
$$

Remark 2.2. (1) At the first look, the differentiation form (2.2) and the integration form (2.4) seem quite different but they are indeed equivalent. To see this, let $f_{2}$ be given in part (2) such that the right-hand side of (2.4) is positive. Take $f_{1}=f_{2}^{\prime} I\left(f_{2}\right)$, then $f_{1}^{\prime}>0$ on $\left(x_{0}, \infty\right)$. Since

$$
f_{1}^{\prime}(x)=-\frac{b(x)}{a(x)} e^{-C(x)} \int_{x}^{\infty} \frac{f_{2}(y) e^{C(y)}}{a(y)} \mathrm{d} y-\frac{f_{2}(x)}{a(x)},
$$


we have $-a f_{1}^{\prime}-b f_{1}=f_{2}$. Hence

$$
\left[-a f_{1}^{\prime}-b f_{1}\right]^{\prime} / f_{1}=f_{2}^{\prime} / f_{1}=I\left(f_{2}\right)^{-1} .
$$

Then (2.2) implies (2.4).

Next, let $f_{1}$ be given in part (1) such that the right-hand side of (2.2) is positive. Fix $p>x_{0}$ and let $c_{1}=f_{1}(p) e^{C(p)}\left(\int_{x_{0}}^{p} a^{-1} e^{C} \mathrm{~d} x\right)^{-1}$. Set $f=c_{1}-a f_{1}^{\prime}-b f_{1}$. Then $f \in C\left[x_{0}, \infty\right) \cap C^{1}\left(x_{0}, \infty\right)$ and $f^{\prime}>0$ on $\left(x_{0}, \infty\right)$. Since

$$
\int_{x_{0}}^{p} \frac{f e^{C}}{a} \mathrm{~d} x=f_{1}\left(x_{0}\right) e^{C\left(x_{0}\right)} \geq 0
$$

we have $f(x)>0$ for $x>p$ and

$$
0<\int_{x_{0}}^{\infty} \frac{f e^{C}}{a} \mathrm{~d} x=f_{1}\left(x_{0}\right) e^{C\left(x_{0}\right)}+c_{1} Z-\lim _{y \rightarrow \infty} f_{1}(y) e^{C(y)} .
$$

Hence $c:=\lim _{y \rightarrow \infty} f_{1}(y) e^{C(y)} \geq 0$ exists and is finite. Now, we set $f_{2}=c / Z-$ $a f_{1}^{\prime}-b f_{1}$. Then $f_{2} \in C\left[x_{0}, \infty\right) \cap C^{1}\left(x_{0}, \infty\right), f_{2}^{\prime}>0$ on $\left(x_{0}, \infty\right)$ and $\pi\left(f_{2}\right)=$ $Z^{-1} \int_{x_{0}}^{\infty} a^{-1} f_{2} e^{C} \mathrm{~d} x=Z^{-1}\left(f_{1} e^{C}\right)\left(x_{0}\right) \geq 0$. Finally, it is easy to see that

$$
I\left(f_{2}\right)^{-1} \geq f_{2}^{\prime} / f_{1}=\left[-a f_{1}^{\prime}-f_{1}\right]^{\prime} / f_{1}
$$

Then (2.4) implies (2.2).

Of course, each of (2.2) and (2.4) has its own advantage. The computation for (2.2) is much easier than (2.4). While, (2.4) is very helpful to see whether the spectral gap is positive or not and to find out an effective test function $f$. The last differential form (2.5) is deduced from (2.4), it is generally weaker than (2.4) and hence weaker than (2.2). But for specific $f,(2.5)$ is not comparable with (2.2). See also Example 2.12 below.

(2) Next, if the function $f$ is the derivative of the eigenfunction corresponding to the first non-trivial eigenvalue $\lambda_{1}=\operatorname{gap}(L)$, then the function $-\left[a f^{\prime}+b f\right]^{\prime} / f$, given on the right-hand side of (2.2), equals $\lambda_{1}$ identically. Conversely, if the function just mentioned is a constant $\alpha>0$ and the function

$$
g(x):=c_{0}+\int_{x_{0}}^{x} f(y) \mathrm{d} y, \quad c_{0}:=-\frac{\left(a f^{\prime}\right)\left(x_{0}\right)}{\alpha}
$$

belongs to $L^{2}(\pi)$ with $f\left(x_{0}\right)=0$ and $\lim _{x \rightarrow \infty} f(x) e^{C(x)}=0$, then $g$ is indeed an eigenfunction (cf. Lemma 6.2) and so the lower bound $\alpha$ given by (2.2) is sharp. In this way, one may construct many examples for which our estimates are exact. Due to the correspondence explained in (1), a similar conclusion holds for the estimate (2.4).

(3) In general, the idea is to regard functions $g$ of the form

$$
c+\int_{x_{0}}^{x} f(y) \mathrm{d} y \quad \text { or } \quad c+\int_{x_{0}}^{x} f^{\prime}(y) I(f)(y) \mathrm{d} y
$$

as an approximation of the eigenfunction. To examine the effectiveness of the approximation, when $g \in L^{2}(\pi)$, simply note by (1.1) that

$$
\operatorname{gap}_{\left[x_{0}, \infty\right)} \leq \frac{1}{\pi\left(g^{2}\right)-(\pi g)^{2}} \int_{x_{0}}^{\infty} a g^{\prime 2} \mathrm{~d} \pi
$$


In the case $g \notin L^{2}(\pi)$, instead of (2.11), we adopt

$$
\operatorname{gap}_{\left[x_{0}, \infty\right)} \leq \underline{\lim }_{n \rightarrow \infty} \frac{1}{\pi_{n}\left(g^{2}\right)-\left(\pi_{n} g\right)^{2}} \int_{x_{0}}^{n} a g^{\prime 2} \mathrm{~d} \pi_{n}
$$

where $\pi_{n}(\mathrm{~d} x)=I_{\left[x_{0}, n\right)}(x) \pi(\mathrm{d} x) / \int_{x_{0}}^{n} \pi(\mathrm{d} y)$ (cf. Lemma 5.1). Furthermore, if $g \in$ $L^{1}(\pi) \backslash L^{2}(\pi)$, then $(2.12)$ becomes

$$
\operatorname{gap}_{\left[x_{0}, \infty\right)} \leq \underline{\lim }_{n \rightarrow \infty} \frac{1}{\pi_{n}\left(g^{2}\right)} \int_{x_{0}}^{n} a g^{\prime 2} \mathrm{~d} \pi_{n}
$$

Clearly, for each test function $f$, we obtain from (2.3) a lower bound for the spectral gap. The correspondence of some elementary functions $f$ and the lower bounds are listed below.

\section{Corollary 2.3.}

(1) $f(x)=\left(c_{1}+x-x_{0}\right)^{\delta}, c_{1} \geq 0, \delta \in \mathbf{R}$.

$$
\operatorname{gap}_{\left[x_{0}, \infty\right)} \geq \begin{cases}\inf _{x>x_{0}}\left[-b^{\prime}(x)-\frac{\delta(\delta-1) a(x)}{\left(c_{1}+x-x_{0}\right)^{2}}-\frac{\delta\left(a^{\prime}+b\right)(x)}{c_{1}+x-x_{0}}\right], & \\ \inf _{x>x_{0}}\left[-b^{\prime}(x)\right] \quad \text { if } \delta=0, & \text { if } \delta=1 . \\ \inf _{x>x_{0}}\left[-b^{\prime}(x)-\frac{\left(a^{\prime}+b\right)(x)}{c_{1}+x-x_{0}}\right] & \text { if }\end{cases}
$$

(2) $f(x)=\left(c_{1}+c_{2}\left(x-x_{0}\right)\right) e^{\delta\left(x-x_{0}\right)}, c_{1}, c_{2} \geq 0, c_{1}+c_{2}>0$ and $\delta \in \mathbf{R}$.

$$
\begin{aligned}
\operatorname{gap}_{\left[x_{0}, \infty\right) \geq \inf _{x>x_{0}}\left[-b^{\prime}(x)-\delta^{2} a(x)-\right.} & \delta\left(a^{\prime}+b\right)(x) \\
& \left.-\frac{c_{2}}{c_{1}+c_{2}\left(x-x_{0}\right)}\left[2 \delta a+a^{\prime}+b\right](x)\right] .
\end{aligned}
$$

(3) $f(x)=c_{1}+c_{2}\left(x-x_{0}\right)+\left(x-x_{0}\right)^{2}, c_{2}>-2 \sqrt{c_{1}}$ or $c_{1}=c_{2}=0$.

$$
\operatorname{gap}_{\left[x_{0}, \infty\right)} \geq \inf _{x>x_{0}}\left[-b^{\prime}(x)-\frac{2 a(x)+\left(a^{\prime}+b\right)(x)\left(c_{2}+2\left(x-x_{0}\right)\right)}{c_{1}+c_{2}\left(x-x_{0}\right)+\left(x-x_{0}\right)^{2}}\right] .
$$

By Corollary 2.3, it is easy to obtain some explicit estimates.

\section{Corollary 2.4.}

(1) If there exist $c_{1}$ and $\varepsilon \leq 1$ (resp. $\varepsilon \geq 1$ ) such that $\left(a^{\prime}+b\right)\left(c_{1}+x-x_{0}\right) \leq \varepsilon a$ (resp. $\left.\left(a^{\prime}+b\right)\left(c_{1}+x-x_{0}\right) \geq \varepsilon a\right)$, then

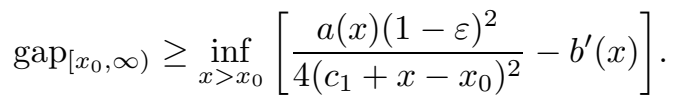

(2) If there exists $\varepsilon_{1}, \varepsilon_{2} \leq 0$ such that $a^{\prime}+b \leq\left(\varepsilon_{1}+\varepsilon_{2}\left(x-x_{0}\right)\right) a$, then $\operatorname{gap}_{\left[x_{0}, \infty\right)} \geq$ $\inf _{x}\left[\left(\frac{\varepsilon_{1}^{2}}{4}-\varepsilon_{2}\right) a(x)-b^{\prime}(x)\right]$.

(3) If $a^{\prime}+b \leq\left(\varepsilon_{1}+\varepsilon_{2}\left(x-x_{0}\right)\right) a$ for some $\varepsilon_{1} \leq-\sqrt{-\varepsilon_{2}}<0$, then $\operatorname{gap}_{\left[x_{0}, \infty\right)} \geq$ $\inf _{x}\left\{-b^{\prime}(x)-2 \varepsilon_{2} a(x)\right\}$.

The next result is deduced from (2.4). Sometimes, it is convenient to decompose the function $f$ given in Theorem $2.1(2)$ as $f=f_{1}+c$ for some $f_{1} \geq 0$ and $c \leq \pi(f)$. 


\section{Corollary 2.5.}

(1) Suppose that $\inf _{x>x_{0}} a(x) /\left(c_{1}+x-x_{0}\right)^{\gamma}:=c>0$ for some $c_{1}>0$ and $\gamma \geq 2$. If there exists $\varepsilon \in(-\infty, \gamma-1)$ such that $\left(c_{1}+x-x_{0}\right) b(x) \leq \varepsilon a(x)$ for large enough $x$ (resp. for all $x \in\left[x_{0}, \infty\right)$ ), then $\operatorname{gap}_{\left[x_{0}, \infty\right)}>0$ (resp. $\left.\operatorname{gap}_{\left[x_{0}, \infty\right)} \geq \frac{c}{4}(\gamma-1-\varepsilon)^{2} c_{1}^{\gamma-2}\right)$. When $\gamma=2$ and $c_{1}=0$, the same conclusion holds by removing the term $c_{1}^{\gamma-2}$.

(2) If there exist some $\varepsilon_{1}$ and $\varepsilon_{2}$, either $\varepsilon_{2}=0$ and $\varepsilon_{1}<0$ or $\varepsilon_{2}<0$, such that $b(x) \leq\left(\varepsilon_{1}+\varepsilon_{2}\left(x-x_{0}\right)\right) a(x)$ for large enough $x$, then $\operatorname{gap}_{\left[x_{0}, \infty\right)}>0$. Furthermore if the condition holds for all $x \in\left[x_{0}, \infty\right)$, then

$$
\begin{aligned}
& \operatorname{gap}_{\left[x_{0}, \infty\right)} \geq \max \left\{\frac{1}{4}\left(\varepsilon_{1} \wedge 0\right)^{2}-\varepsilon_{2}, \quad-\varepsilon_{2}\left[1+\int_{0}^{\infty} e^{u+\varepsilon_{2} u^{2} /\left(2\left(\varepsilon_{1} \vee 0\right)^{2}\right)} \mathrm{d} u\right]^{-1},\right. \\
& \left.\frac{1}{4}\left[\int_{0}^{\infty} e^{\varepsilon_{1} u+\varepsilon_{2} u^{2} / 2} \mathrm{~d} u\right]^{-2}\right\} \inf _{x} a(x) .
\end{aligned}
$$

(3) If

$$
c_{1}:=\sup _{x>x_{0}} e^{-C(x)} \int_{x}^{\infty} \frac{e^{C(u)}}{a(u)} \mathrm{d} u<\infty
$$

and

$$
c_{2}:=\sup _{x>x_{0}} e^{-C(x)} \int_{x}^{\infty} e^{C(u)} \mathrm{d} u<\infty
$$

then $\operatorname{gap}_{\left[x_{0}, \infty\right)} \geq 1 /\left(4 c_{1} c_{2}\right)$.

(4) If $c:=\sup _{x>x_{0}} \frac{a(x)}{e^{C(x)}} \int_{x}^{\infty} \frac{e^{C(u)}}{a(u)} \mathrm{d} u<\infty$, then $\operatorname{gap}_{\left[x_{0}, \infty\right)} \geq \inf _{x} a(x) /\left(4 c^{2}\right)$. In particular, if $\lim _{x \rightarrow \infty} e^{C(x)} / a(x)=0$ and $\varlimsup_{x \rightarrow \infty} a(x) /\left[a^{\prime}(x)-b(x)\right]<\infty$, then $\operatorname{gap}_{\left[x_{0}, \infty\right)}>0$.

(5) If $b \equiv 0$, then $\operatorname{gap}_{\left[x_{0}, \infty\right)} \geq\left\{4 \sup _{x>x_{0}}\left(x-x_{0}\right) \int_{x}^{\infty} a(u)^{-1} \mathrm{~d} u\right\}^{-1}$.

Observe that it is usually not difficult to find a test function so that the estimates $(2.2)$ and (2.4) are non-trivial out of a local region. That is, replacing " $x>x_{0}$ " with " $x>N$ " for large enough $N$, we obtain a positive lower bound. For instance, if $a(x) \equiv 1$, then the function $f(x)=\exp [-\varepsilon C(x)],(\varepsilon \in(0,1))$ works for $(2.4)$ out of a local region. Next, if $\inf _{x>N}\left[-b^{\prime}(x)\right]>0$, then the function $f(x)=x$ is enough for (2.2) out of a local region. We are now going to show that this is indeed sufficient for a non-trivial estimate since we can always modify the test function so that the infimum over the whole space $\left[x_{0}, \infty\right)$ is positive. Besides, the results given below actually provide us some optimizing methods to improve the resulting estimate.

Corollary 2.6. Given $f \in C^{1}\left[x_{0}, \infty\right)$ with $\pi(f) \geq 0, f^{\prime}(x)>0$ for large enough $x$ and

$$
\varlimsup_{x \rightarrow \infty} I(f)(x)<\infty
$$

Then, we have

$$
\operatorname{gap}_{\left[x_{0}, \infty\right)} \geq \sup _{c>0} \inf _{x>x_{0}} I\left(f_{1}\right)(x)^{-1}>0
$$

where $f_{1}(x)=c x /(1+x)+f(x)$. 
This corollary is deduced from (2.4) by using $f_{1}$ instead of the original $f$. The additional term $c x /(1+x)$ changes the sign of $f^{\prime}$ locally but without interfering with the convergence in (2.14). The next corollary is quite convenient in practice since the test function is fixed and it is also very effective if the decay of the drift $b(x)$ is not slower than linear.

To state the result, we need some notations which will be used several times in what follows. Let $K \in C\left(x_{0}, D\right)$ be a non-decreasing function so that $\left(x-x_{0}\right) K(x) / a(x)$ is locally integrable. Define

$$
\begin{aligned}
F^{r}(s) & =\int_{x_{0}}^{s \wedge r} \frac{u-x_{0}}{a(u)}[K(r)-K(u)] \mathrm{d} u, \quad r \in\left(x_{0}, D\right) . \\
\delta(K) & =\sup _{r \in\left(x_{0}, D\right)} K(r) \inf _{s \in\left(x_{0}, r\right]} \frac{\left(s-x_{0}\right) \exp \left[-F^{r}(s)\right]}{\int_{x_{0}}^{s} \exp \left[-F^{r}(u)\right] \mathrm{d} u} .
\end{aligned}
$$

Then, we have

$$
\delta(K) \geq \sup _{r \in\left(x_{0}, D\right)} K(r) \exp \left[-F^{r}(r)\right]=K\left(r_{0}\right) \exp \left[-1+\int_{x_{0}}^{r_{0}} \frac{\left(u-x_{0}\right) K(u)}{a(u)} \mathrm{d} u\right],
$$

where $r_{0}$ is the unique solution to the equation

$$
K(r)=\left(\int_{x_{0}}^{r} \frac{u-x_{0}}{a(u)} \mathrm{d} u\right)^{-1}, \quad r \in\left(x_{0}, D\right) .
$$

When $D<\infty$ and (2.19) has no solution in $(0, D)$, we set $r_{0}=D$.

Corollary 2.7. Choose a non-decreasing function $K \in C\left(x_{0}, \infty\right)$ such that

$$
K(r) \leq \inf _{x \geq r}\left[-\left(a^{\prime}+b\right)(x) /\left(x-x_{0}\right)-b^{\prime}(x)\right]+\sup _{y} b^{\prime}(y)
$$

$\left(\right.$ resp. $\left.K(r) \leq \inf _{x>r}\left[-b(x) /\left(x-x_{0}\right)\right]\left(r>x_{0}\right)\right)$. Assume that $\left(x-x_{0}\right) K(x) / a(x)$ is locally integrable. Then, we have

$$
\operatorname{gap}_{\left[x_{0}, \infty\right)} \geq \beta_{0}+\delta(K),
$$

where $\beta_{0}=-\sup _{x} b^{\prime}(x)\left(\right.$ resp. $\left.\beta_{0}=0\right)$.

The following examples illustrate the power of the above results. Here, we consider the half line $[0, \infty)$ only.

Example 2.8. Take $b(x)=-b(b>0), a(x) \equiv a$. By Corollary 2.3 (2) with $\delta=b /(2 a)$, we get $\operatorname{gap}_{[0, \infty)} \geq b^{2} /(4 a)$ which is sharp (see [6, example 1.10]). Corollary 2.4 (2) or Corollary $2.5(2)$ with $\varepsilon_{1}=-b / a$ and $\varepsilon_{2}=0$ as well as Corollary 2.5 (5) give us the same bound.

Example 2.9. Take $a(x) \equiv 1$ and $b(x)=-\alpha x^{\beta},(\alpha>0, \beta>-1)$. Applying Corollary 2.6 to $f(x)=\exp \left[\varepsilon x^{\beta+1}\right](\varepsilon<\alpha /(\beta+1))$, it follows that $\operatorname{gap}_{[0, \infty)}>0$. To get some explicit bounds, we apply Corollary 2.7 which is available iff $\beta \geq 1$. The linear case $(\beta=1)$ will be treated in the next example. We now assume that $\beta>1$. Then, the lower bounds provided by Corollary 2.7 and (2.18) for the two choices of $K$ are

$$
2^{\frac{\beta-1}{\beta+1}}[\alpha(\beta+1)]^{\frac{2}{\beta+1}} \exp \left[-1+\frac{2}{\beta+1}\right] \text { and } 2^{\frac{\beta-1}{\beta+1}} \alpha^{\frac{2}{\beta+1}} \exp \left[-1+\frac{2}{\beta+1}\right]
$$


respectively. Clearly, the first bound is bigger than the second one. However, if we consider $a(x)=\left(1+x^{2}\right)^{2}$ and $b(x)=-\alpha x^{3}$, then the alternative choice of $K$ works for all $\alpha>0$ but the first choice of $K$ works only for $\alpha>1$. Therefore, the two choices of $K$ in Corollary 2.7 are not comparable.

Example 2.10. Take $b(x)=-\alpha x(\alpha>0), a(x) \equiv 1$. By Corollary 2.3 (1) with $c_{1}=0$ and $\delta=1$ (or Corollary 2.4 (2) with $\varepsilon_{1}=0$ and $\varepsilon_{2}=-\alpha$, or Corollary $2.7)$, we get $\operatorname{gap}_{[0, \infty)} \geq 2 \alpha$. This estimate is sharp since $g(x)=x^{2} / 2-1 /(2 \alpha)$ is an eigenfunction and so Remark $2.2(2)$ is suitable. The same bound can be obtained by using (2.4) or (2.5) with $f(x)=x^{2}-1 / \alpha$.

Example 2.11. Take $b(x)=-\alpha x-\beta$. Then

$$
V(x)=-\left(\log a(x)+\int_{0}^{x}(\alpha r+\beta) a(r)^{-1} \mathrm{~d} r\right) .
$$

If (2.1) holds, by Corollary 2.3 (1) with $\delta=0$, it follows that $\operatorname{gap}_{[0, \infty)} \geq \alpha^{+}$. This provides us a non-trivial lower bound for a large number of concrete examples since $a(x)$ is quite arbitrary. If we take $a(x) \equiv 1$, then, Corollary 2.4 (3) gives us $\operatorname{gap}_{[0, \infty)} \geq 3 \alpha$ provided $\beta \geq \sqrt{\alpha}>0$. Moreover, in the case that $\alpha=\beta=1$, the estimate is indeed sharp by Remark $2.2(2)$. This is quite interesting since the change of $\beta$ from 0 to 1 leads to not only the change of the spectral gap from 2 to 3 but also the change of the eigenfunction from quadratic to cubic. We now consider the particular case that $a(x)=(1+x)^{2}$ and $\beta=\alpha$. Then $V(x)=$ $-(2+\alpha) \log (1+x)$ and (2.1) holds iff $\alpha>-1$. By Corollary 2.4 (1) or applying (2.3) to the function $f(x)=(1+x)^{(\alpha-1) / 2}$ or applying (2.4) to $f(x)=(1+x)^{(\alpha+1) / 2}$, we obtain $\operatorname{gap}_{[0, \infty)} \geq(\alpha+1)^{2} / 4 \geq \alpha^{+}$. The last equality holds iff $\alpha=1$. Note that when $\alpha>1$, even though $g(x):=x+1$ is in $L^{2}(\pi)$ and satisfies $a g^{\prime \prime}+b g^{\prime}=-\alpha g$, but this $g$ is still not the eigenfunction of $\lambda_{1}$ since $g^{\prime}(0) \neq 0$. For general $\alpha>-1$, the function $g(x):=(1+x)^{(\alpha+1) / 2}$ satisfies $a g^{\prime \prime}+b g^{\prime}=-(\alpha+1)^{2} g / 4$ but $g$ is not the eigenfunction of $\lambda_{1}$ since $g \notin L^{2}(\pi)$. Thus, Remark $2.2(2)$ is not suitable for this example. However, applying $(2.13)$ to $g(x)=(1+x)^{(\alpha+1) / 2}$, we obtain

$$
\operatorname{gap}_{[0, \infty)} \leq \underline{\lim }_{n \rightarrow \infty} \frac{\int_{0}^{n} a g^{\prime 2} e^{V} \mathrm{~d} x}{\int_{0}^{n} g^{2} e^{V} \mathrm{~d} x} \leq \underline{\lim }_{n \rightarrow \infty} \frac{a(n) g^{\prime}(n)^{2} e^{V(n)}}{g(n)^{2} e^{V(n)}}=\frac{(1+\alpha)^{2}}{4} .
$$

We have thus achieved the exact bound. This example shows that in order to attain the sharp estimate, we do have some freedom of the choice of test functions rather than using the eigenfunction only.

Example 2.12. Take $b(x) \equiv 0$ and $a(x)=(1+x)^{\alpha}$. Obviously, (2.1) holds iff $\alpha>1$. By Corollary 2.4 (1) with $c_{1}=1$ and $\varepsilon=\alpha$ or by Corollary 2.5 (1) with $\gamma=\alpha$ and $c_{1}=1$, we get $\operatorname{gap}_{[0, \infty)} \geq(\alpha-1)^{2} / 4$ for all $\alpha \geq 2$. This is similar to the last example. Next, applying $(2.13)$ to $g(x)=(1+x)^{(\alpha-1) / 2}$, we obtain $\operatorname{gap}_{[0, \infty)}=0$ for all $\alpha \in(1,2)$, which is the same as the lower bound given by Theorem 2.1. Therefore, gap $[0, \infty)>0$ iff $\alpha \geq 2$ and our estimate is sharp for all $\alpha \leq 2$. However, the lower bound $(\alpha-1) / 2$ is not sharp when $\alpha>2$. To see this, applying (2.4) to the family $\left\{f(x)=(1+x)^{\varepsilon}-(\alpha-1) /(\alpha-1-\varepsilon): \varepsilon>0\right\}$, we get

$$
\begin{aligned}
\operatorname{gap}_{[0, \infty)} & \geq \sup _{\varepsilon \in(0, \alpha-2)}\left[(\alpha-1-\varepsilon)(\alpha-2+\varepsilon)\left(\frac{\alpha-2+\varepsilon}{\alpha-2}\right)^{(\alpha-2) / \varepsilon}\right] \\
& \geq e(\alpha-1)(\alpha-2) .
\end{aligned}
$$


Setting $\varepsilon=1 / 2$ and then letting $\alpha \downarrow 2$, the first estimate of (2.20) gives us $\operatorname{gap}_{[0, \infty)} \geq 1 / 4$, which is sharp. We will show in the next section (Example 3.6) that the principal term $e \alpha^{2}$ of the lower bound is also exact as $\alpha \rightarrow \infty$. Applying (2.2) to the family $\left\{f(x)=(1+x)^{\varepsilon}: \varepsilon>0\right\}$, we obtain $\operatorname{gap}_{[0, \infty)} \geq(\alpha-1)^{2} / 4$. As for (2.5), we get gap $[0, \infty) \geq 1 / 4$ (independent of $\alpha$ ). Replacing $f$ with $f-\pi(f)$, $\pi(f)=(\alpha-1) /(\alpha-1-\varepsilon)$, it follows from $(2.5)$ that

$$
\begin{aligned}
\operatorname{gap}_{[0, \infty)} & \geq \sup _{\varepsilon \in(0,1 \wedge(\alpha-2))}\left[(1-\varepsilon)(\alpha-2+\varepsilon)\left(\frac{\pi(f)(\alpha-2+\varepsilon)}{\alpha-2}\right)^{(\alpha-2) / \varepsilon}\right] \\
& \geq e^{2-1 /(\alpha-1)}(\alpha-2) .
\end{aligned}
$$

All these estimates are exact at $\alpha=2$. From these, we see that (2.5) is weaker than (2.4) but it is not comparable with (2.2) for the specific functions.

Example 2.13. Take $a(x)=(1+x)^{3}$ and $b(x)=(1+x)^{2}$. By Corollary $2.4(1)$ or Corollary $2.5(1)$, we have gap $[0, \infty) \geq 1 / 4$. On the other hand, applying (2.4) to $f(x)=\log (1+x)-1$, we get $\operatorname{gap}_{[0, \infty)} \geq \inf _{x>0} \frac{1+x}{\log (1+x)}=e$.

\section{The Case of the Full Line}

Set $C(x)=\int_{0}^{x} b(u) a(u)^{-1} \mathrm{~d} u$. Then " $Z<\infty$ " becomes

$$
\int_{-\infty}^{\infty} \frac{e^{C(x)}}{a(x)} \mathrm{d} x<\infty
$$

The process is non-explosive iff

$$
\min \left\{\int_{0}^{\infty} \mathrm{d} x e^{-C(x)} \int_{0}^{x} \frac{e^{C(y)}}{a(y)} \mathrm{d} y, \int_{-\infty}^{0} \mathrm{~d} x e^{-C(x)} \int_{x}^{0} \frac{e^{C(y)}}{a(y)} \mathrm{d} y\right\}=\infty .
$$

Intuitively, the idea in this section is to divide the full line into two half lines. However, there are some technical problems. Note that the spectral gap for the full line can not be bigger than the maximum of the ones for the half lines. Thus, the test function $f$ must be connected in some way around the reference point $x_{0}$. For instance, in order for the approximating function $g$ of the eigenfunction to be in $C^{2}(\mathbf{R})$, we require that $f \in C^{1}(\mathbf{R})$ in the first term below and $f \in C(\mathbf{R})$ with $f\left(x_{0}\right)=0$ in the second term below. Actually, what we have in mind is taking the reference point $x_{0}$ to be the place at which the eigenfunction vanishes, even though the precise place is usually unknown in advance.

As a variation of $I(f)$, define

$$
I^{-}(f)(x)=\frac{e^{-C(x)}}{f^{\prime}(x)} \int_{x}^{-\infty} \frac{f(u) e^{C(u)}}{a(u)} \mathrm{d} u, \quad x<x_{0} .
$$

Theorem 3.1. Assume that (3.1) and (3.2) hold. Let $x_{0} \in \mathbf{R}$.

(1) For every function $f \in C^{2}(\mathbf{R})$ with $f(x)>0$ for all $x$, we have

$$
\begin{aligned}
\operatorname{gap}(L) & \geq \inf _{x}\left[\left(-a f^{\prime}-b f\right)^{\prime} / f\right](x) \\
& =\inf _{x}\left[-b^{\prime}-\left[a f^{\prime \prime}+\left(a^{\prime}+b\right) f^{\prime}\right] / f\right](x) .
\end{aligned}
$$

(2) Let $C(x)=\int_{x_{0}}^{x} a^{-1} b$. For every function $f \in C(\mathbf{R}) \cap C^{1}\left(\mathbf{R} \backslash\left\{x_{0}\right\}\right) \cap L^{1}(\pi)$ with $f\left(x_{0}\right)=0, f^{\prime}(x)>0$ for all $x \neq x_{0}$, we have

$$
\operatorname{gap}(L) \geq\left(\delta_{1} \vee \delta_{2}\right)^{-1},
$$


where

$$
\delta_{1}=\sup _{x>x_{0}} I(f)(x), \quad \delta_{2}=\sup _{x<x_{0}} I^{-}(f)(x) .
$$

In particular, if moreover $f \in C^{2}(\mathbf{R})$, then

$$
\operatorname{gap}(L) \geq \inf _{x \neq x_{0}}\left[-a f^{\prime \prime}-b f^{\prime}\right](x) / f(x) .
$$

Applying (3.4) to the functions $f(x)=c_{1}+\left|x-x_{0}\right|^{\delta}\left(c_{1} \geq 0, \delta=0\right.$ or $\left.\delta>1\right)$ and $f(x)=e^{\delta\left(x-x_{0}\right)}(\delta \in \mathbf{R})$, we obtain the following result.

\section{Corollary 3.2.}

(1) If there exists $\varepsilon \leq 1$ (resp. $\varepsilon \geq 1$ ) such that $\left(a^{\prime}+b\right)\left(x-x_{0}\right) \leq \varepsilon a$ (resp. $\left.\left(a^{\prime}+b\right)\left(x-x_{0}\right) \geq \varepsilon a\right)$, then $\operatorname{gap}(L) \geq \inf _{x \neq x_{0}}\left[\frac{a(x)(1-\varepsilon)^{2}}{4\left(x-x_{0}\right)^{2}}-b^{\prime}(x)\right]$.

(2) If there exists $\varepsilon \leq 0$ such that $a^{\prime}+b \leq \varepsilon a$, then $\operatorname{gap}(L) \geq \inf _{x}\left[\frac{\varepsilon^{2}}{4} a(x)-b^{\prime}(x)\right]$.

\section{Corollary 3.3.}

(1) Suppose that $\inf _{x \neq x_{0}} a(x) /\left(x-x_{0}\right)^{2}:=c>0$. If there exists $\varepsilon<1$ such that $\left(x-x_{0}\right) b(x) \leq \varepsilon a(x)$ for large $|x|$ (resp. for all $\left.x \neq x_{0}\right)$, then $\operatorname{gap}(L)>0$ $\left(\right.$ resp. $\left.\left.\operatorname{gap}(L) \geq \frac{c}{4}(1-\varepsilon)^{2}\right)\right)$.

(2) If there exist some $\varepsilon_{1}$ and $\varepsilon_{2}$, either $\varepsilon_{2}=0$ and $\varepsilon_{1}<0$ or $\varepsilon_{2}<0$, such that $\operatorname{sgn}\left(x-x_{0}\right) b(x) \leq\left(\varepsilon_{1}+\varepsilon_{2}\left|x-x_{0}\right|\right) a(x)$ for large enough $|x|$, then $\operatorname{gap}(L)>0$. Furthermore if the condition holds for all $x \neq x_{0}$, then

$$
\operatorname{gap}(L) \geq \frac{1}{4}\left[\int_{0}^{\infty} e^{\varepsilon_{1} u+\varepsilon_{2} / 2} \mathrm{~d} u\right]^{-2} \inf _{x} a(x) .
$$

(3) If

In particular, if $\varepsilon_{2}=0$, then $\operatorname{gap}(L) \geq \frac{1}{4} \varepsilon_{1}^{2} \inf _{x} a(x)$.

$$
\begin{array}{ll}
c_{1}:=\sup _{x>x_{0}} e^{-C(x)} \int_{x}^{\infty} \frac{e^{C(u)}}{a(u)} \mathrm{d} u, & c_{1}^{-}:=\sup _{x<x_{0}} e^{-C(x)} \int_{-\infty}^{x} \frac{e^{C(u)}}{a(u)} \mathrm{d} u, \\
c_{2}:=\sup _{x>x_{0}} e^{-C(x)} \int_{x}^{\infty} e^{C(u)} \mathrm{d} u, & c_{2}^{-}:=\sup _{x<x_{0}} e^{-C(x)} \int_{-\infty}^{x} e^{C(u)} \mathrm{d} u
\end{array}
$$

(4) If

are all finite, then $\operatorname{gap}_{\left[x_{0}, \infty\right)} \geq 1 / \max \left\{4 c_{1} c_{2}, 4 c_{1}^{-} c_{2}^{-}\right\}$.

$$
c:=\max \left\{\sup _{x>x_{0}} \frac{a(x)}{e^{C(x)}} \int_{x}^{\infty} \frac{e^{C(u)}}{a(u)} \mathrm{d} u, \sup _{x<x_{0}} \frac{a(x)}{e^{C(x)}} \int_{-\infty}^{x} \frac{e^{C(u)}}{a(u)} \mathrm{d} u\right\}<\infty,
$$

then $\operatorname{gap}(L) \geq \inf _{x} \frac{a(x)}{4 c^{2}}$. In particular, if $\lim _{|x| \rightarrow \infty} e^{C(x)} / a(x)=0$ and $\varlimsup_{|x| \rightarrow \infty} a(x) /\left[a^{\prime}(x)-b(x)\right]<\infty$, then $\operatorname{gap}(L)>0$.

(5) If $b \equiv 0$, then

$$
\operatorname{gap}(L) \geq \frac{1}{4}\left[\max \left\{\sup _{x>x_{0}}\left(x-x_{0}\right) \int_{x}^{\infty} a(u)^{-1} \mathrm{~d} u, \sup _{x<x_{0}}\left(x_{0}-x\right) \int_{-\infty}^{x} a(u)^{-1} \mathrm{~d} u\right\}\right]^{-1} .
$$

Part (3) and (4) of the corollary improve respectively the first two parts of [6, Theorem 1.3] which were proved by using an analytic approach rather than the coupling one. Moreover, the present proof becomes very simple. 
By adding a new term, $c \tan ^{-1}(x)$ or $c x / \sqrt{1+x^{2}}$ for instance, to the original function $f$, from Theorem 3.1 (2), we obtain the following result.

Corollary 3.4. Suppose that there exists a function $f \in C(\mathbf{R}) \cap C^{1}\left(\mathbf{R} \backslash\left\{x_{0}\right\}\right)$ with $f\left(x_{0}\right)=0, f^{\prime}(x)>0$ for all large enough $|x|$ and

$$
\max \left\{\varlimsup_{x \rightarrow+\infty} I(f)(x), \quad \varlimsup_{x \rightarrow-\infty} I^{-}(f)(x)\right\}<\infty .
$$

Then, we have $\operatorname{gap}(L)>0$.

Corollary 3.5. Choose $K \in C\left(\mathbf{R} \backslash\left\{x_{0}\right\}\right)$ such that $K(x)$ is non-decreasing as $\left|x-x_{0}\right|$ increases, moreover, $K(r) \leq \inf _{x \geq r} b(x) /\left(x_{0}-x\right)$ for all $r \geq x_{0}$ and $K(r) \leq \inf _{x \leq r} b(x) /\left(x_{0}-x\right)$ for all $r \leq x_{0}$. Assume that $\left(x-x_{0}\right) K(x) / a(x)$ is locally integrable. Define $F^{r}(s)$ as in (2.16) for $x_{0} \leq s \leq r$ or $r \leq s \leq x_{0}$ and then define $\delta(K)$ as in (2.17) with $D=\infty$. Next, define $\delta^{-}(K)$ in the same way but replacing " $r>x_{0}$ " and " $s \in\left(x_{0}, r\right]$ " with " $r<x_{0}$ " and " $s \in\left[r, x_{0}\right)$ " respectively. Then, we have $\operatorname{gap}(L) \geq \delta(K) \wedge \delta^{-}(K)$.

We are now ready to mention a nice result due to Kac and Krein [11] and Kotani [12] by using a different approach: Let $b \equiv 0$. Then

$$
\frac{1}{4} \delta^{-1} \leq \operatorname{gap}_{[0, \infty)} \leq \delta^{-1}, \quad \frac{1}{4}\left(\delta \vee \delta^{-}\right)^{-1} \leq \operatorname{gap}(L) \leq\left(\delta \vee \delta^{-}\right)^{-1},
$$

where $\delta=\sup _{x \geq 0} x \int_{x}^{\infty} a(u)^{-1} \mathrm{~d} u$ and $\delta^{-}=\sup _{x \leq 0} x \int_{x}^{-\infty} a(u)^{-1} \mathrm{~d} u$. Clearly, the lower bounds coincide with Corollary 2.5 (5) and Corollary 3.3 (5) respectively. To illustrate the power of (3.8), it suffices to look at an example with the half line.

Example 3.6. Consider the Example 2.12 again. Then, by (3.8), we have $\delta^{-1}=1$ if $\alpha=2$ and

$$
\delta^{-1}=\frac{(\alpha-1)^{\alpha}}{(\alpha-2)^{\alpha-2}}=(\alpha-1)^{2}\left(1+\frac{1}{\alpha-2}\right)^{\alpha-2} \sim e \alpha^{2} \quad \text { if } \alpha>2 .
$$

Combining this with the lower bound given in Example 2.12, we see that the upper bound here has the correct order as $\alpha \rightarrow \infty$ and the lower bound is exact when $\alpha=2$.

The examples given below not only illustrate the use of the our results but also show some difference between the half line and the full line.

Example 3.7. Take $b(x)=-\alpha x-\beta$. If (3.1) and (3.2) hold, then as in Example 2.11 , we have $\operatorname{gap}(L) \geq \alpha^{+}$. When $\alpha>0$ and $\beta=0$, we indeed have $\operatorname{gap}(L)=\alpha$ for every $a(x)$ having the properties: symmetric with respect to the origin, satisfying (3.1) and (3.2) and $\int x^{2} \mathrm{~d} \pi<\infty$, since then $g(x)=x$ is an eigenfunction of $\lambda_{1}=\alpha$. Especially, when $a(x) \equiv 1$, we have gap $(L)=\alpha$ but not $2 \alpha$ given in Example 2.10.

Example 3.8. Consider the special case of the above example, $b(x)=-\alpha x$ and $a(x)=1+x^{2}$. Then, $C(x)=-\frac{\alpha}{2} \log \left(1+x^{2}\right)$ and (3.1) holds iff $\alpha>-1$. We have just seen that $\operatorname{gap}(L)=\alpha$ for all $\alpha>1$. This is different from Example 2.12. Next, applying Theorem 3.1 (2) to the test function $f(x)=x\left(1+x^{2}\right)^{\varepsilon}, \varepsilon=(\alpha-1) / 4$, we obtain

$$
\begin{aligned}
\delta_{1}=\delta_{2} & =\sup _{x>0} \frac{\left(1+x^{2}\right)^{\alpha / 2}}{\left(1+x^{2}\right)^{\varepsilon}+2 \varepsilon x^{2}\left(1+x^{2}\right)^{\varepsilon-1}} \int_{x}^{\infty} \frac{u\left(1+u^{2}\right)^{-\alpha / 2+\varepsilon}}{1+u^{2}} \mathrm{~d} u \\
& =\sup _{x>0} \frac{1+x^{2}}{\left[1+(1+2 \varepsilon) x^{2}\right](\alpha-2 \varepsilon)} \leq \frac{1}{(1+2 \varepsilon)(\alpha-2 \varepsilon)}=\frac{4}{(1+\alpha)^{2}} .
\end{aligned}
$$


Finally, applying (2.12), we get

$$
\begin{aligned}
\operatorname{gap}(L) & \leq \lim _{n \rightarrow \infty} \frac{\int_{-n}^{n} a(x) f^{\prime}(x)^{2} e^{V(x)} \mathrm{d} x}{\int_{-n}^{n} f(x)^{2} e^{V(x)} \mathrm{d} x} \\
& =\lim _{n \rightarrow \infty} \frac{\int_{-n}^{n}\left(1+x^{2}\right)^{-\alpha / 2}\left[\left(1+x^{2}\right)^{\varepsilon}+2 \varepsilon x^{2}\left(1+x^{2}\right)^{-1+\varepsilon}\right]^{2}}{\int_{-n}^{n} x^{2}\left(1+x^{2}\right)^{-1-\alpha / 2+2 \varepsilon} \mathrm{d} x} \\
& =\frac{(1+\alpha)^{2}}{4} .
\end{aligned}
$$

Therefore, $\operatorname{gap}(L)=(\alpha+1)^{2} / 4$ for all $\alpha \in(-1,1]$.

Example 3.9. Take $b(x)=-\alpha x^{3}(\alpha>0)$ and $a(x)=\left(1+x^{2}\right)^{2}$. Applying (3.7) to $f(x)=x\left(1+x^{2}\right)^{-1 / 2}$, we obtain $\operatorname{gap}(L) \geq 3$ which is independent of $\alpha$. On the other hand, by Corollary 3.5 with $x_{0}=0, K(r)=\alpha r^{2}$ and $r_{0}^{2}=(\sqrt{2 \alpha+1}+1) / \alpha$, we obtain $K\left(r_{0}\right)=\sqrt{2 \alpha+1}+1$ and

$$
\operatorname{gap}(L) \geq K\left(r_{0}\right)\left(1+K\left(r_{0}\right) / \alpha\right)^{\alpha / 2} \exp \left[-1-\frac{\alpha K\left(r_{0}\right)}{2\left(\alpha+K\left(r_{0}\right)\right)}\right] \succsim \sqrt{2 \alpha+1} e^{-1 / 2} .
$$

Especially, when $\alpha=4$, then the first bound equals $16 e^{-2} \approx 2.1654$.

Example 3.10. Take $a(x) \equiv 1$ and $b(x)=-x+\cos x$. This is clearly a perturbation of the ordinary O.U.-process. However, when we apply (3.4) to $f(x) \equiv 1$, which gives the exact eigenvalue of the O.U.-process, we get the trivial bound. We now adopt a comparison technique (see also Proposition 4.5). Note that

$$
C(u)-C(x)=-u^{2} / 2+x^{2} / 2+\sin u-\sin x \leq-u^{2} / 2+x^{2} / 2+2 .
$$

Inserting this into (3.6) with $f(x)=x$, it follows that $\operatorname{gap}(L) \geq e^{-2}$. The estimate can be further improved by noticing

$$
C(u)-C(x) \leq-u^{2} / 2+x^{2} / 2+\varepsilon \sin u-\varepsilon \sin x+2(1-\varepsilon)
$$

and using $f(x)=x+\varepsilon \cos x$ instead of $f(x)=x$. Then we obtain gap $(L) \geq(2 e)^{-1}$ by setting $\varepsilon=1 / 2$.

To conclude this section, we mention some examples for which the eigenfunction $g \in C^{2}(\mathbf{R}) \cap L^{2}(\pi)$ but non-linear.

Examples 3.11. Let $a(x) \equiv 1$. Then we have gap $(L)=1$ for the following choices of $b(x)$.

(1) $g(x)=x\left(c+x^{2}\right), c>0 . b(x)=-\frac{x}{3}\left[1+\frac{2(9+c)}{3 x^{2}+c}\right]$.

(2) $g(x)=\int_{0}^{x} e^{c y^{2 n}} \mathrm{~d} y, n \in \mathbf{Z}_{+}, c \in \mathbf{R} . b(x)=-2 n c x^{2 n-1}-e^{-c x^{2 n}} \int_{0}^{x} e^{c y^{2 n}} \mathrm{~d} y$.

(3) $g(x)=c x+\sin x, c>1 . b(x)=-\frac{c x}{c+\cos x}$.

To prove the assertion, simply use (3.4) with $f=g^{\prime}$ and note that both $g$ and $b$ are odd functions. 


\section{The General Case}

In contrast to the cases of the half line or full line, the structure of the eigenfunction of $\lambda_{1}$ in the higher dimensional case is too complex to be understood and it is often not monotone with respect to the ordinary semi-order. Here, a diffusion semigroup $P_{t}$ is said to be monotone if $P_{t} f(x) \leq P_{t} f(y)$ holds for all $x \leq y$ and all monotone (non-decreasing) continuous functions $f$. Even in the case that the eigenfunction is monotone, one still requires the process to be monotone which is a quite strong restrictive condition especially for the higher dimensional diffusions (refer to [8] for details). Thus, in general, it is not practical to use the eigenfunction or its approximation as the distance we required and so we should adopt a different strategy. Roughly speaking, our goal is as follows. First, we use the coupling method on some simple distances in $\mathbf{R}^{d}$ and reduce our problem to the case of the half line. Then, applying the idea given in Section 2 we construct a new distance $f \circ d$ for some suitable function $f$. Fortunately, in this way, we still obtain good enough estimates for the spectral gap.

Let $\widetilde{L}$ be a coupling operator of $L, d(x, y)$ be a distance which is in $C^{2}$ away from the set $\left\{(x, x): x \in \mathbf{R}^{d}\right\}$ and let $D=\sup _{x, y} d(x, y)$. Then there exist two functions $A$ and $B$ on $\mathbf{R}^{d} \times \mathbf{R}^{d}$ such that for each $f \in C^{2}[0, D)$ (refer to [4]),

$$
\widetilde{L} f \circ d(x, y)=A(x, y) f^{\prime \prime}(d(x, y))+B(x, y) f^{\prime}(d(x, y)), \quad x \neq y .
$$

Note that $\widetilde{L}$ is a degenerate elliptic operator on $\mathbf{R}^{d} \times \mathbf{R}^{d}$, we have $A(x, y) \geq 0$ for all $x$ and $y$. One key step of the coupling approach is to find a function $f \in C^{2}[0, D)$ with $f(0)=0, f^{\prime}>0$ and $f^{\prime \prime} \leq 0$ on $[0, D)$ such that

$$
\widetilde{L} f \circ d(x, y) \leq-\delta f \circ d(x, y), \quad x \neq y,
$$

for some constant $\delta>0$. Next, choose functions $\alpha, \beta \in C(0, D)$ such that

$$
\alpha(r) \leq \inf _{d(x, y)=r} A(x, y), \quad \beta(r) \geq \sup _{d(x, y)=r} B(x, y) .
$$

Then, for (4.2), it suffices that

$$
\alpha(r) f^{\prime \prime}(r)+\beta(r) f^{\prime}(r) \leq-\delta f(r), \quad r \in(0, D) .
$$

We have thus reduced (4.2) to (4.4). Denote by $\lambda^{*}$ the largest constant $\delta$ in (4.2) as $f$ varies. Clearly, $\lambda^{*}$ dominates the largest $\delta$ in (4.4). The next result is parallel to Theorem 2.1 .

\section{Theorem 4.1.}

(1) For every function $f \in C^{2}[0, D)$ with $f(0)=0, f^{\prime}>0$ and $f^{\prime \prime} \leq 0$ on $[0, D)$, we have

$$
\lambda^{*} \geq \inf _{r \in(0, D)}\left[\left(-\alpha f^{\prime \prime}-\beta f^{\prime}\right) / f\right](r) .
$$

(2) Define $C(r)=\int_{0}^{r} \alpha^{-1} \beta$ and then define $I(f)$ as in (1.2) but replacing a $(u)$ and $[0, \infty)$ with $\alpha(u)$ and $[0, D)$ respectively. For every function $f \in C[0, D) \cap$ $L^{1}(\pi)$ with $\pi(f) \geq 0$ on $(0, D)$ and satisfying

$$
f(r) \geq-\beta(r) e^{-C(r)} \int_{r}^{D} \frac{f(u) e^{C(u)}}{\alpha(u)} \mathrm{d} u,
$$


we have

$$
\lambda^{*} \geq \inf _{r \in(0, D)}\left\{f(r)^{-1} \int_{0}^{r} \mathrm{~d} s e^{-C(s)} \int_{s}^{D} \frac{f(u) e^{C(u)}}{\alpha(u)} \mathrm{d} u\right\}^{-1},
$$

In particular, if moreover $f \in C^{1}(0, D), f(0)=0$ and $f^{\prime}>0$ on $(0, D)$, then

$$
\lambda^{*} \geq \inf _{r \in(0, D)} I(f)(r)^{-1} .
$$

Theorem 4.1 is also meaningful for diffusion processes on a manifold which will be treated in a separate paper. Next, if there exists a coupling such that $\inf _{x, y} A(x, y)>0$ and $\lambda^{*}>0$, then the $L$-diffusion process is ergodic. Part (1) of Theorem 4.1 is rather simple but it has the following useful consequence, which is an analog of the alternative choice of Corollary 2.7.

Corollary 4.2. Choose a non-decreasing function $K \in C(0, D)$ such that $K(r) \leq$ $\inf _{s \in[r, D)}[-\beta(s) / s], r \in(0, D)$. Assume that $r K(r) / \alpha(r)$ is locally integrable on $(0, D)$. Define $F^{r}(s)$ as in (2.16) and then define $\delta(K)$ in $(2.17)$ with $x_{0}=0$. Then, we have $\lambda^{*} \geq \delta(K)$.

Remark 4.3. (1) The condition (4.6) is used for the non-positive property of the second derivative of the function required by (4.2) or (4.4). However, when $A(x, y)$ in (4.1) is indeed a function of $d(x, y)$ only and $\alpha(r)$ is taken to be the common value of $A(x, y)$ when $d(x, y)=r$, we do not need (4.6). In this case, the resulting function $f \circ d$ may not be a distance but this does not interfere with our proof.

(2) When $\beta(r) \geq 0$ on $(0, D)$, the condition (4.6) is trivial. In the case of $\beta(r)<0$ on $(0, D)$ and $\lim _{r \rightarrow D} f(r) e^{-C(r)} / \beta(r)=0,(4.6)$ can be rewritten as follows:

$$
\int_{r}^{D}\left(\frac{f}{\beta}\right)^{\prime}(u) e^{C(u)} \mathrm{d} u \geq 0 .
$$

More simply,

$$
f^{\prime} \beta-\beta^{\prime} f \geq 0 \quad \text { on } \quad(0, D)
$$

is enough for (4.9).

By virtue of (4.8), part (2) of Theorem 2.1 and its Corollaries 2.6 and 2.7 are available with a slight modification. We omit the details here to save space. The reason why we use $\lambda^{*}$ here rather than $\operatorname{gap}(L)$ is the following. Our approach requires that the eigenfunction be Lipschitz with respect to the distance we adopted. In the compact case, this is not a problem. But for the non-compact case, this may not be true. To overcome this difficulty, we adopt a localizing procedure [6], which then yields some technical problems. So, in general, we are still unable to claim that $\lambda^{*}$ is indeed a lower bound of $\operatorname{gap}(L)$. However, the conclusion holds for the one-dimensional case.

Corollary 4.4. When $d=1$, Theorem 4.1 and Corollary 4.2 hold if $\lambda^{*}$ is replaced by $\operatorname{gap}(L)$.

Before moving further, we mention a simple comparison result which is a direct consequence of (1.1) (refer to [6] and [16]).

Proposition 4.5. (1) Let $\bar{L} \sim(\bar{a}, V)$, if $a(x)-\bar{a}(x) \geq 0$ for all $x$, then

$$
\operatorname{gap}(L) \geq \operatorname{gap}(\bar{L}) .
$$


(2) Let $\bar{L} \sim(a, \bar{V})$, we have

$$
\operatorname{gap}(L) \geq \operatorname{gap}(\bar{L}) \exp [-\delta(V-\bar{V})],
$$

where $\delta(f)=\sup f-\inf f$.

Let us also mention a sufficient condition for the regularity of Dirichlet forms. In general, [9, Theorem 1.6.3] says that the semi-group is recurrent if there exists $\left\{u_{n}\right\} \subset C_{0}^{\infty}\left(\mathbf{R}^{d}\right)$ such that $u_{n} \rightarrow 1$ and $\lim _{n \rightarrow \infty} \int\left\langle a \nabla u_{n}, \nabla u_{n}\right\rangle \mathrm{d} \pi=0$. From this we conclude that the Dirichlet form is regular if there exists $r_{n} \uparrow \infty$ such that

$$
\lim _{n \rightarrow \infty} \int_{r_{n} \leq|x| \leq r_{n}+1} \operatorname{tr} a(x) \mathrm{d} \pi=0 .
$$

Actually, choose $h \in C^{\infty}(\mathbf{R})$ such that $\left\|h^{\prime}\right\|_{\infty} \leq 2$ and $h(r)=1$ for $r \leq 0, h(r)=0$ for $r \geq 1$. Take $u_{n}(x)=h\left(|x|-r_{n}\right)$, then $u_{n} \rightarrow 1$ and (4.13) implies $\int\left\langle a \nabla u_{n}, \nabla u_{n}\right\rangle \mathrm{d} \pi$ $\rightarrow 0$.

To study the spectral gap of diffusions in $\mathbf{R}^{d}$, we consider three concrete distances: the Euclidean distance, the $L_{1}$-distance and the Riemannian distance induced by a positive definite diagonal matrix which is dominated by $a(x)$. To state the result, we need some notations. Choose positive functions $a_{i} \in C^{2}\left(\mathbf{R}^{d}\right)(i \leq d)$ such that $a-\operatorname{diag}\left\{a_{1}, a_{2}, \cdots, a_{d}\right\} \geq 0$ (non-negative definite) and $\inf _{i, x} a_{i}(x)>0$. Let $\bar{b}_{i}=a_{i} \partial_{i} V+\partial_{i} a_{i}(i \leq d)$. Next, set $\alpha_{2}=4$ and choose $\alpha_{1}, \alpha_{3} \in C\left(\mathbf{R}_{+}\right)$such that

$$
\begin{aligned}
& 0<\alpha_{1}(r) \leq \inf _{|x-y|=r}\left\{\min _{i}\left(\sqrt{a_{i}(x)}-\sqrt{a_{i}(y)}\right)^{2}+4 \min _{i} \sqrt{a_{i}(x) a_{i}(y)}\right\}, \\
& 0<\alpha_{3}(r) \leq \inf _{|x-y|_{1}=r}\left\{\sum_{i=1}^{d}\left(\sqrt{a_{i}(x)}-\sqrt{a_{i}(y)}\right)^{2}+4 \min _{i} \sqrt{a_{i}(x) a_{i}(y)}\right\},
\end{aligned}
$$

where $|\cdot|$ is the ordinary Euclidean norm and $|x-y|_{1}=\sum_{i=1}^{d}\left|x_{i}-y_{i}\right|$. Next, choose $\beta_{j}(j=1,2,3)$ as follows.

(1) Put $\sigma=\sqrt{\operatorname{diag}\left\{a_{1}, a_{2}, \cdots, a_{d}\right\}}$ and choose $\beta_{1} \in C(0, \infty)$ so that

$$
\begin{array}{r}
\beta_{1}(r) \geq \sup _{|x-y|=r}|x-y|^{-1}\left\{\|\sigma(x)-\sigma(y)\|^{2}-|x-y|^{-2}|(\sigma(x)-\sigma(y))(x-y)|^{2}\right. \\
+\langle\bar{b}(x)-\bar{b}(y), x-y\rangle\} .
\end{array}
$$

(2) If $a_{i}(x)$ depends on $x_{i}$ only for all $i$. Set

$$
\rho(x, y)=\left[\sum_{i=1}^{d}\left(\int_{x_{i}}^{y_{i}} a_{i}(r)^{-1 / 2} \mathrm{~d} r\right)^{2}\right]^{1 / 2}, \quad D=\sup _{x, y} \rho(x, y),
$$

and $h_{i}=\sqrt{a_{i}} \partial_{i} V+\partial_{i} \sqrt{a_{i}}, i \leq d$. Choose $\beta_{2} \in C(0, D)$ so that

$$
\beta_{2}(r) \geq \sup _{\rho(x, y)=r} \rho(x, y)^{-1} \sum_{i=1}^{d}\left[h_{i}(y)-h_{i}(x)\right] \int_{x_{i}}^{y_{i}} a_{i}(r)^{-1 / 2} \mathrm{~d} r, \quad r \in(0, D) .
$$

(3) If $a_{i}(x)$ depends on $x_{i}$ only and $\bar{b}_{i}(x)$ is non-decreasing in $x_{k}$ for $k \neq i$. Choose $\beta_{3} \in C(0, \infty)$ so that

$$
\beta_{3}(r) \geq \sup _{x \geq y,|x-y|_{1}=r} \sum_{i=1}^{d}\left[\bar{b}_{i}(x)-\bar{b}_{i}(y)\right], \quad r>0 .
$$


Finally choose non-decreasing functions $K_{1}, K_{3} \in C(0, \infty)$ and $K_{2} \in C(0, D)$ so that $K_{j}(r) \leq \inf _{s \geq r}\left[-\beta_{j}(s) / s\right]$.

Theorem 4.6. Theorem 4.1 and Corollary 4.2 are valid if the functions $\alpha, \beta, K$ and $\lambda^{*}$ are replaced by $\alpha_{j}, \beta_{j}, K_{j}$ and $\operatorname{gap}(L)$ respectively for each $j=1,2,3$.

Let $a(x)=\alpha(x) \sigma^{2}$ for some positive $\alpha \in C^{2}\left(\mathbf{R}^{d}\right)$ and positive definite matrix $\sigma$. To use Theorem 4.6, by Proposition 4.5, one may compare $a(x)$ with a diagonal matrix directly. But, as was pointed out in [3], [6], the result should be better if we directly use the distance $\left|\sigma^{-1}(x-y)\right|$ instead of the Euclidean one. To this end, take the coordinate transformation $y=\sigma^{-1} x$. Then $\partial / \partial x_{i}=\sum_{k=1}^{d}\left(\sigma^{-1}\right)_{i k} \partial / \partial y_{k}, i \leq d$, and the operator $L \sim(a, b)$ becomes

$$
L^{(y)}=\alpha(\sigma y) \sum_{k=1}^{d} \frac{\partial^{2}}{\partial y_{k}^{2}}+\sum_{k=1}^{d}\left(\sum_{i=1}^{d} b_{i}(\sigma y)\left(\sigma^{-1}\right)_{i k}\right) \frac{\partial}{\partial y_{k}}
$$

which is in the desired form of Theorem 4.6.

The following result simplifies the form of $K_{j}$ 's given above. It can be considered as an extension of [7, Theorem 1.3] to multidimensional diffusion processes in the context of spectral gap.

Corollary 4.7. Let $a_{i}, \bar{b}_{i}$ and $\alpha_{j}$ be the same as in Theorem 1.1. Suppose that $a_{i}(x)$ depends only on $x_{i}$ for all $i$. Set $\kappa=\max _{i}\left\|\nabla \sqrt{a_{i}}\right\|_{\infty}^{2}$. Fix a point $p \in \mathbf{R}^{d}$ and let $\lambda_{\min }(A)$ be the smallest real part of eigenvalues of matrix A. According to the three cases in Theorem 4.6, we define $\theta_{j}(j=1,2,3)$ as follows.

(1) $\theta_{1}(r)=\inf _{|x-p| \geq r} \lambda_{\min }\left(-\partial_{j} \bar{b}_{i}(x)\right), \quad r \geq 0$.

(2) $\theta_{2}(r)=\inf _{\rho(x, p) \geq r} \lambda_{\min }\left(-X_{i} X_{j} \bar{V}(x)\right)$, where $X_{i}=\sqrt{a_{i}\left(x_{i}\right)} \partial_{i}$ and $\bar{V}=V+$ $\log \sqrt{a_{1} \cdots a_{d}}$.

(3) If $\bar{b}_{i}(x)$ is non-decreasing in $x_{k}$ for $i \neq k$, let $\theta_{3}(r)=\inf _{|x-p|_{1} \geq r}\left[-\max _{j} \sum_{i} \partial_{j} \bar{b}_{i}(x)\right]$.

Next, define $\left.\gamma_{j}(r)=r^{-1} \int_{0}^{r} \theta_{j}(u) \mathrm{d} u(j=1,2,3), K_{1}(r)=\gamma_{1}(r / 2)-\left(1-d^{-1}\right) \kappa, K_{3}(r)\right)$ $=\gamma_{3}(r / 2)$ for $r>0$ and $K_{2}(r)=\gamma_{2}(r / 2)$ for $r \in(0, D)$. Then, Corollary 4.2 holds for these $K_{j}$ 's with the same replacements made in Theorem 4.6. In particular, if $\max \left\{\theta_{1}(\infty)-\kappa\left(1-d^{-1}\right), \theta_{2}(D), \theta_{3}(\infty)\right\}>0$, then we have $\operatorname{gap}(L)>0$. Here, $\theta_{i}(\infty)(i=1,3)$ and $\theta_{2}(D)$ are understood as the limits as $r \rightarrow \infty$ and $r \rightarrow D$ respectively.

Obviously, when $d=1$, the case of $j=1$ coincides with the case of $j=3$ for both Theorem 4.6 and Corollary 4.7. As for $d>1$, the first may be better than the latter. For example, this is the case for $d=2, a=I$ and $V(x)=-\frac{1}{2} x_{1}^{2}+x_{1} x_{2}-x_{2}^{2}$. Conversely, the latter may be better if $\left\|\nabla a_{i}\right\|_{\infty}$ is large for some $i \leq d$. From these and Example 4.8 below, we conclude that the cases of $j=1,2,3$ are not comparable to each other.

Example 4.8. Consider Example 3.9 again. We have $V(x)=\alpha / 2-\alpha /\left[2\left(1+x^{2}\right)\right]-$ $(2+\alpha / 2) \log \left(1+x^{2}\right)$ and $(4.13)$ holds. For $r>0$, we have $\inf _{x}(b(x+r)-b(x))=$ $-\alpha r^{3} / 4$. Take $K_{1}(r)=\alpha r^{2} / 4$ and $\alpha_{1}(r)=4$, then $F_{1}^{r}(r)=\alpha r^{4} / 64$. By Theorem 4.6 with $j=1$ or 3 , we obtain $\operatorname{gap}(L) \geq \sqrt{2 \alpha} \exp [-1 / 2]$. This is weaker but close to $(3.9)$. 
Next, we have $\bar{V}(x)=-\alpha /\left[2\left(1+x^{2}\right)\right]-(1+\alpha / 2) \log \left(1+x^{2}\right)$. Let $X(x)=$ $\left(1+x^{2}\right) \frac{\mathrm{d}}{\mathrm{d} x}$, then

$$
-X^{2} \bar{V}(x)=(2+\alpha)\left(1+x^{2}\right)-\frac{2 \alpha}{1+x^{2}}+\alpha \geq 2, \quad x \in \mathbf{R} .
$$

By Corollary 4.7 (2) we obtain $\operatorname{gap}(L) \geq 2$ which is independent of $\alpha$.

Example 4.9. The lower bound $\operatorname{gap}(L) \geq \alpha^{+}$given in Example 3.7 can be also obtained by using Corollary 4.7 with $j=1$ or 3 .

Example 4.10. We now return to Example 3.10. Let $h(r)=-\sup _{x}[b(y)-b(x)], r$ $\geq 0$. It is easy to check that $h(2 \pi+r)-2 \pi-r=h(r)-r$ and $h(r)=r-2 \sin \frac{r}{2}$ for $r \in[0,2 \pi]$. Since $r^{-1} h(r)$ is increasing, it can be taken as $K_{1}(r)$. By Theorem 4.6 we have

$$
\operatorname{gap}(L) \geq \sup _{r \in[0, \pi]}\left(1-r^{-1} \sin r\right) \exp [\cos r+(r \sin r) / 2-1] .
$$

By setting $r=1.95$, we obtain $\operatorname{gap}(L) \geq 0.329$.

Example 4.11. When $a=I$ we take $\alpha=4$ and then the estimate provided by (4.7) with $f(r) \equiv 1$ coincides with the one obtained by using the first moment of the coupling time (refer to [6]). Note that the test function $f(x) \equiv 1$ can not be allowed for (4.8) since $f^{\prime} \equiv 0$. But (4.8) does often produce better estimates. Especially, take $d=1$ and $b(x)=-4 x^{3}$. Choose $\beta(r)=-r^{3}$. By (4.7) we get the lower bound $[\Gamma(5 / 4)+1 / 8)]^{-1} \approx 0.9695$ which is the same as in [6, Example 1.9]. Nevertheless, applying (4.8) to the test function $f(x)=\log (1+x)$ and noticing Remark 4.3 (1), a numerical computation shows that $\operatorname{gap}(L) \geq 2.4395$.

Example 4.12. Take $a=I$ and $b_{i}(x)=\sum_{j} b_{i j} x_{j}$, where $\left(b_{i j}\right)$ is symmetric with $b_{i j} \geq 0$ for $i \neq j$ and $\sum_{i} b_{i j}=-1$ for all $j$. Next, take $g(x)=\sum_{i} x_{i}$. We have

$$
V(x)=\frac{1}{2} \sum_{i, j=1}^{d} b_{i j} x_{i} x_{j} \leq \frac{1}{2} \sum_{i=1}^{d}\left(b_{i i} x_{i}^{2}+\frac{1}{2} \sum_{j \neq i} b_{i j}\left(x_{i}^{2}+x_{j}^{2}\right)\right)=-\frac{1}{2}|x|^{2} .
$$

Thus (4.13) holds and $g \in L^{2}(\pi)$. Moreover, it is easy to check that $\pi(g)=0$. Hence $\operatorname{gap}(L)=1$ which is just the lower bound provided by Corollary 4.7 with $j=3$.

To conclude this section, we study the Poincaré inequality with respect to the absolute distribution of the process generated by $L$. This provides a new way to estimate $\operatorname{gap}(L)$ and may be useful in the study of the spectral gap on path space. The idea used here comes from [10] and [17] in which the logarithmic Sobolev inequalities on path space were studied for diffusions over a Riemannian manifold.

Theorem 4.13. Suppose that there exists $\bar{a}>0$ such that $\langle a(x) u, u\rangle \leq \bar{a}|u|^{2}$ for all $x, u \in \mathbf{R}^{d}$. Let $a(x)=\sigma(x) \sigma(x)^{*}$ and set

$$
K=\sup _{x \neq y}|x-y|^{-2}\left[\|\sigma(x)-\sigma(y)\|^{2}+\langle b(x)-b(y), x-y\rangle\right] .
$$

We have

$$
P_{t} f^{2}(x) \leq K^{-1} \bar{a}(\exp [2 K t]-1) P_{t}|\nabla f|^{2}(x)+\left(P_{t} f(x)\right)^{2}
$$

for all $x \in \mathbf{R}^{d}, t \geq 0$ and $f \in C^{1}\left(\mathbf{R}^{d}\right)$ with $P_{t} f^{2}(x)<\infty$. When $K=0$, the coefficient on the right-hand side is understood as the limit as $K \rightarrow 0$. 
We mention that (4.14) can be sharp. For example, take $L=\Delta$, then $2 t$ is the smallest constant so that (4.14) holds. The bounded assumption of $a$ is unnatural, due to the limitation of the present proof, but we do not know how to remove it.

Remark 4.14. The process considered in Theorem 4.13 is not necessarily reversible. Next, the $L$-diffusion process is ergodic if $K<0$. Then, by letting $t \rightarrow \infty$ in (4.14), we obtain

$$
\operatorname{gap}(L) \geq-K \bar{a}^{-1} \inf _{x} \lambda_{\min }(a(x))
$$

\section{Preparations for the Proofs}

Lemma 5.1. Suppose that $D_{n} \uparrow \mathbf{R}^{d}$ is a sequence of normal domains and let $\operatorname{gap}\left(D_{n}\right)$ denote the first Neumann eigenvalue of $L$ on $D_{n}$, then we have $\operatorname{gap}(L) \geq$ $\varlimsup_{n \rightarrow \infty} \operatorname{gap}\left(D_{n}\right)$. When $d=1$, we indeed have $\operatorname{gap}\left(D_{n}\right) \downarrow \operatorname{gap}(L)$.

Proof. a) Note that (refer to [1] and [15])

$$
\operatorname{gap}\left(D_{n}\right)=\inf \left\{\pi_{n}(\langle a \nabla f, \nabla f\rangle): f \in C^{1}\left(D_{n}\right), \pi_{n}(f)=0, \pi_{n}\left(f^{2}\right)=1\right\},
$$

where $\pi_{n}(f)=\pi\left(I_{D_{n}} f\right)$. For any $\varepsilon>0$, choose $f \in C^{1}\left(\mathbf{R}^{d}\right)$ such that $\pi(f)=$ $0, \pi\left(f^{2}\right)=1$ and $\pi(\langle a \nabla f, \nabla f\rangle) \leq \operatorname{gap}(L)+\varepsilon$. Then, there exists $n_{0} \geq 1$ such that

$$
\int_{D_{n}}\left(f-\int_{D_{n}} f \mathrm{~d} \pi\right)^{2} \mathrm{~d} \pi \geq 1-\varepsilon, \quad n \geq n_{0} .
$$

Hence

$$
\operatorname{gap}\left(D_{n}\right) \leq \frac{\int_{D_{n}}\langle a \nabla f, \nabla f\rangle \mathrm{d} \pi}{\int_{D_{n}}\left(f-\int_{D_{n}} f \mathrm{~d} \pi\right)^{2} \mathrm{~d} \pi} \leq \frac{\operatorname{gap}(L)+\varepsilon}{1-\varepsilon} .
$$

b) Next, when $d=1$, we need only to prove that $\operatorname{gap}_{\left[p_{1}, q_{1}\right]} \geq \operatorname{gap}_{\left[p_{2}, q_{2}\right]} \geq \operatorname{gap}(L)$ for $\left[p_{1}, q_{1}\right] \subset\left[p_{2}, q_{2}\right]$. Let $u$ be an eigenfunction with respect to $\operatorname{gap}_{\left[p_{1}, q_{1}\right]}$, then $u^{\prime}\left(p_{1}\right)=u^{\prime}\left(q_{1}\right)=0$. We extend $u$ to $\mathbf{R}$ by setting $u(r)=u\left(p_{1}\right)$ for $r \leq p_{1}$ and $u(r)=u\left(q_{1}\right)$ for $r \geq q_{1}$. Then $u \in C^{1}(\mathbf{R})$, by (5.1) we obtain $\operatorname{gap}_{\left[p_{2}, q_{2}\right]} \leq \operatorname{gap}_{\left[p_{1}, q_{1}\right]}$.

c) If in addition (4.13) holds, there exist non-negative functions $u_{n} \in C_{0}^{\infty}(\mathbf{R})$ such that $u_{n} \uparrow 1$ and $\int a\left(u_{n}^{\prime}\right)^{2} \mathrm{~d} \pi \rightarrow 0$ as $n \rightarrow \infty$. By (1.1) together with an approximation argument, we have

$$
\operatorname{gap}(L) \leq \lim _{n \rightarrow \infty} \frac{\int a\left[\left(u u_{n}\right)^{\prime}\right]^{2} \mathrm{~d} \pi}{\int\left(u u_{n}\right)^{2} \mathrm{~d} \pi-\left(\int u u_{n} \mathrm{~d} \pi\right)^{2}} \leq \operatorname{gap}_{\left[p_{1}, q_{1}\right]} .
$$

d) To avoid the use of the sufficient condition (4.13), take $V_{\varepsilon}=V-\varepsilon a, Z_{\varepsilon}=$ $\int e^{V_{\varepsilon}} \mathrm{d} x, \varepsilon>0$. Then $V_{\varepsilon} \uparrow V$ and $Z_{\varepsilon} \uparrow Z$ as $\varepsilon \downarrow 0$. Let $L_{\varepsilon} \sim\left(a, V_{\varepsilon}\right)$, then $L_{\varepsilon}$ satisfies (4.13). By (1.1) we have

$$
\operatorname{gap}(L) \leq \underline{\lim }_{\varepsilon \rightarrow 0} \operatorname{gap}\left(L_{\varepsilon}\right) \leq \operatorname{gap}_{[p, q]} .
$$

Suppose that $a(x)=\sigma(x) \sigma(x)^{*}$ for all $x$. Let $\widetilde{L} \sim(\tilde{a}, \tilde{b})$ be the operator of the coupling by reflection [4]:

$$
\tilde{a}(x, y)=\left(\begin{array}{cc}
a(x) & c(x, y) \\
c(x, y)^{*} & a(y)
\end{array}\right), \quad \tilde{b}(x, y)=\left(\begin{array}{l}
b(x) \\
b(y)
\end{array}\right),
$$

where $c(x, y)=\sigma(x)\left(I-2 \bar{u} \bar{u}^{*}\right) \sigma(y)^{*}$ and $\bar{u}=(x-y) /|x-y|$. 
Lemma 5.2. Let $S=\prod_{i=1}^{d}\left[p_{i}, q_{i}\right]$ and $\left(x_{t}, y_{t}\right)$ be the coupling by reflection of the reflecting $L$-diffusion process on $S$. If $\sigma_{i j}=\delta_{i j} \sigma_{i i}\left(x_{i}\right)$ and $b_{i}(x)$ is non-decreasing in $x_{k}$ for $k \neq i$, then the coupling preserves the ordinary semi-order: $x_{0} \geq y_{0}$ implies $P^{x_{0}, y_{0}}\left(x_{t} \geq y_{t}, t \geq 0\right)=1$.

Proof. One may compare the conditions of the lemma with the criteria given in [8]. Let $T=\inf \left\{t \geq 0: x_{t}=y_{t}\right\}$ be the coupling time, then we need only to prove the order-preservation up to time $T$.

a) For $n \geq 1$, choose $C_{n} \in C(\mathbf{R})$ with $\operatorname{supp} C_{n} \subset\left(0, n^{-1}\right), 0 \leq C_{n} \leq 2 n$, and $\int C_{n}(u) \mathrm{d} u=1$. Define $\phi_{n}(r)=\int_{0}^{r} \mathrm{~d} s \int_{0}^{s} C_{n}(u) \mathrm{d} u$, then $0 \leq \phi_{n}^{\prime}(r) \leq 1,0 \leq \phi_{n}^{\prime \prime}(r) \leq$ $2 /\left(n r^{2}\right)$ and $\phi_{n}(r) \uparrow r^{+}$. Next, note that $b_{i}(x)$ is non-decreasing in $x_{k}$ for $k \neq i$ and $\phi_{n}^{\prime}\left(y_{i}-x_{i}\right)=0$ for $y_{i} \leq x_{i}$, we have

$$
\begin{aligned}
\widetilde{L} \phi_{n}\left(y_{i}-x_{i}\right)= & \phi_{n}^{\prime}\left(y_{i}-x_{i}\right)\left(b_{i}(y)-b_{i}(x)\right) \\
& +\phi_{n}^{\prime \prime}\left(y_{i}-x_{i}\right)\left[\sum_{j=1}^{d}\left(\sigma_{j j}\left(y_{j}\right)-\sigma_{j j}\left(x_{j}\right)\right)^{2}\right. \\
& \left.\quad+\frac{4}{|x-y|^{2}} \sum_{j=1}^{d}\left(y_{j}-x_{j}\right)^{2} \sigma_{j j}\left(x_{j}\right) \sigma_{j j}\left(y_{j}\right)\right] \\
\leq & N \sum_{j=1}^{d}\left(y_{i}-x_{i}\right)^{+}+\frac{N}{n \varepsilon^{2}}
\end{aligned}
$$

for some constant $N$ and all $x, y \in S$ with $|x-y| \geq \varepsilon>0$. Let $L_{i+}^{(1)}, L_{i-}^{(1)}$ be the local times of $x_{t}$ on $\left\{x_{i}=q_{i}\right\},\left\{x_{i}=p_{i}\right\}$ respectively, and let $L_{i+}^{(2)}, L_{i-}^{(2)}$ be those of $y_{t}$. Note that $\phi_{n}^{\prime}\left(y_{i}-x_{i}\right)=0$ for $y_{i} \leq x_{i}$ and

$$
I_{\left[q_{i}-\varepsilon, q_{i}\right]}\left(x_{i}\right) \leq I_{\left[q_{i}-\varepsilon, q_{i}\right]}\left(y_{i}\right), \quad I_{\left[p_{i}, p_{i}+\varepsilon\right]}\left(y_{i}\right) \leq I_{\left[p_{i}, p_{i}+\varepsilon\right]}\left(x_{i}\right)
$$

for $q_{i} \geq y_{i} \geq x_{i} \geq p_{i}$. We have

$$
\begin{aligned}
& \int_{t_{1}}^{t_{2}} \phi_{n}^{\prime}\left(y_{i}(s)-x_{i}(s)\right) \mathrm{d}\left(L_{i+}^{(1)}(s)+L_{i-}^{(2)}(s)-L_{i+}^{(2)}(s)-L_{i-}^{(1)}(s)\right) \\
& =\lim _{\varepsilon \rightarrow 0} \int_{t_{1}}^{t_{2}} \phi_{n}^{\prime}\left(y_{i}(s)-x_{i}(s)\right)\left(I_{\left[q_{i}-\varepsilon, q_{i}\right]}\left(x_{i}(s)\right)-I_{\left[q_{i}-\varepsilon, q_{i}\right]}\left(y_{i}(s)\right)\right. \\
& \left.\quad+I_{\left[p_{i}, p_{i}+\varepsilon\right]}\left(y_{i}(s)\right)-I_{\left[p_{i}, p_{i}+\varepsilon\right]}\left(x_{i}(s)\right)\right) \mathrm{d} s \leq 0, \quad t_{1} \leq t_{2} .
\end{aligned}
$$

b) Let $T_{\varepsilon}=\inf \left\{t \geq 0:\left|x_{t}-y_{t}\right| \leq \varepsilon\right\}$, by (5.2) and (5.3) we have

$$
\begin{aligned}
& E^{x_{0}, y_{0}} \sum_{i=1}^{d}\left[\phi_{n}\left(y_{i}\left(t_{2} \wedge T_{\varepsilon}\right)-x_{i}\left(t_{2} \wedge T_{\varepsilon}\right)\right)-\phi_{n}\left(y_{i}\left(t_{1} \wedge T_{\varepsilon}\right)-x_{i}\left(t_{1} \wedge T_{\varepsilon}\right)\right)\right] \\
& \leq E^{x_{0}, y_{0}} \int_{t_{1} \wedge T_{\varepsilon}}^{t_{2} \wedge T_{\varepsilon}} \sum_{i=1}^{d} \widetilde{L} \phi_{n}\left(y_{i}(t)-x_{i}(t)\right) \mathrm{d} t \\
& \leq\left(t_{2}-t_{1}\right) \frac{d N}{n \varepsilon^{2}}+N \int_{t_{1}}^{t_{2}} E^{x_{0}, y_{0}} \sum_{i=1}^{d}\left(y_{i}\left(t \wedge T_{\varepsilon}\right)-x_{i}\left(t \wedge T_{\varepsilon}\right)\right)^{+} \mathrm{d} t, \quad t_{1} \leq t_{2} .
\end{aligned}
$$


By letting $n \rightarrow \infty$, we obtain

$$
\frac{d}{d t} E^{x_{0}, y_{0}} \sum_{i=1}^{d}\left(y_{i}\left(t \wedge T_{\varepsilon}\right)-x_{i}\left(t \wedge T_{\varepsilon}\right)\right)^{+} \leq N E^{x_{0}, y_{0}} \sum_{i=1}^{d}\left(y_{i}\left(t \wedge T_{\varepsilon}\right)-x_{i}\left(t \wedge T_{\varepsilon}\right)\right)^{+} .
$$

Therefore

$$
E^{x_{0}, y_{0}} \sum_{i=1}^{d}\left(y_{i}\left(t \wedge T_{\varepsilon}\right)-x_{i}\left(t \wedge T_{\varepsilon}\right)\right)^{+}=0
$$

which implies $P^{x_{0}, y_{0}}\left(y_{i}\left(t \wedge T_{\varepsilon}\right) \leq x_{i}\left(t \wedge T_{\varepsilon}\right)\right)=1, \quad t \geq 0$. Since $T_{\varepsilon} \uparrow T$ as $\varepsilon \downarrow 0$, the lemma follows by letting $\varepsilon \rightarrow 0$.

The following result summarizes our approach to estimate gap $(L)$ by using coupling.

Theorem 5.3. Let $D_{n} \uparrow \mathbf{R}^{d}$ be a sequence of normal domains with inward normal vector fields $V_{n}$ of $\partial D_{n}$ under the Riemannian metric $\left(g\left(\partial_{i}, \partial_{j}\right)\right)=a^{-1}$. Next, let $d(x, y): \mathbf{R}^{d} \times \mathbf{R}^{d} \rightarrow[0, \infty)$ be in $C^{2}$ out of $\left\{(x, x): x \in \mathbf{R}^{d}\right\}$ and having the properties: $d(x, y)=0$ iff $x=y$, for each $n$ and $x \in D_{n},\left.V_{n} d(x, \cdot)(y)\right|_{\partial D_{n}} \leq 0$ and there exists $c_{n}>0$ such that $d(x, y) \geq c_{n}|x-y|$ for $x, y \in D_{n}$. If there exists a coupling operator $\widetilde{L}$ of $L$ such that $\widetilde{L} d(x, y) \leq-\delta d(x, y)$ for some $\delta>0$ and all $x \neq y$, then $\operatorname{gap}(L) \geq \delta$.

Proof. Fix $n \geq 1$, let $\left(x_{t}, y_{t}\right)$ be the reflecting $\widetilde{L}$-diffusion process on $D_{n} \times D_{n}$ under the Riemannian metric $\left(g\left(\partial_{i}, \partial_{j}\right)\right)=a^{-1}$. Let $L_{t}$ be the local time of the process on $\partial\left(D_{n} \times D_{n}\right)$, then

$$
\begin{aligned}
\mathrm{d} d\left(x_{t}, y_{t}\right) & =\mathrm{d} M_{t}+\widetilde{L} d\left(x_{t}, y_{t}\right) \mathrm{d} t+\left(V_{n} d\left(\cdot, y_{t}\right)\left(x_{t}\right)+V_{n}\left(x_{t}, \cdot\right)\left(y_{t}\right)\right) \mathrm{d} L_{t} \\
& \leq \mathrm{d} M_{t}-\delta d\left(x_{t}, y_{t}\right) \mathrm{d} t
\end{aligned}
$$

up to the coupling time $T$ for some martingale $M_{t}$. Here, we take $V_{n} f(x)=0$ for $x \notin \partial\left(D_{n}\right)$. By Lemma 5.2, [15, Lemma 2.4] and [3, Theorem 6.2] we obtain $\operatorname{gap}\left(D_{n}\right) \geq \delta$ which proves Theorem 5.3.

\section{Proofs of Theorem 2.1 And its Corollaries}

Proof of Theorem 2.1. a) Let $f \in C^{1}\left[x_{0}, \infty\right) \cap C^{2}\left(x_{0}, \infty\right)$ with $f>0$ on $\left(x_{0}, \infty\right)$ and define $g(x)=\int_{x_{0}}^{x} f$. Then $g$ is strictly increasing and so $d(x, y):=|g(x)-g(y)|$ is a distance in $\left[x_{0}, \infty\right)$. Because the process is monotone (see [8]), we simply use the classical coupling: $\widetilde{L} h(x, y)=(L h(\cdot, y))(x)+(L h(x, \cdot))(y)$ for all $h \in C^{2}\left(\left[x_{0}, \infty\right)^{2}\right)$ and $x \neq y$. Then

$$
\begin{aligned}
\widetilde{L} d(x, y) & =\widetilde{L} d(x, \cdot)(y)-\widetilde{L} d(\cdot, y)(x)=L g(y)-L g(x) \\
& \leq-d(x, y) \inf _{y>x}\left[\left(-\left(a g^{\prime \prime}+b g^{\prime}\right)(y)+\left(a g^{\prime \prime}+b g^{\prime}\right)(x)\right) /(g(y)-g(x))\right] \\
& \leq-d(x, y) \inf _{z>x_{0}}\left[\left(-a f^{\prime}-b f\right)^{\prime} / f\right](z), \quad x \leq y .
\end{aligned}
$$

Here in the last step, we have used the Mean Value Theorem. Part (1) of Theorem 2.1 then follows from Theorem 5.3.

b) For part (2) of Theorem 2.1, the proof is similar but applying the coupling to the function

$$
g(x)=\int_{x_{0}}^{x} f^{\prime}(y) I(f)(y) \mathrm{d} y
$$


To prove $(2.5)$, note that

$$
\left(f^{\prime} e^{C}\right)^{\prime}=\left(a f^{\prime \prime}+b f^{\prime}\right) e^{C} / a .
$$

By assumption, we have $-\left(f^{\prime} e^{C}\right)^{\prime} \geq c f e^{C} / a$. Therefore, $I(f)(x) \leq c^{-1}$ and so the conclusion follows from (2.4). It remains to check that $g^{\prime}>0$ on $\left(x_{0}, \infty\right)$. But this holds iff $\pi(f) \geq 0$ due to the fact that $f^{\prime}>0$ on $\left(x_{0}, \infty\right)$.

Proof of Corollary 2.4. The assertions follow from that of Corollary 2.3 correspondingly with the specific parameters given below.

(1) $\delta=(1-\varepsilon) / 2$.

(2) $c_{1}=0, c_{2}=-\varepsilon_{2}$ and $\delta=-\varepsilon_{1} / 2$.

(3) $c_{1}=\left(\varepsilon_{2}+\varepsilon_{1}^{2}\right) / \varepsilon_{2}^{2}, c_{2}=2 \varepsilon_{1} / \varepsilon_{2}$.

To prove Corollary 2.5, we need a simple result which is an extension of $[6$, Lemma 3.1].

Lemma 6.1. Let $m \in C\left(\left[x_{0}, \infty\right) ; \mathbf{R}_{+}\right)$and $n \in C\left(\left[x_{0}, \infty\right) ;(0, \infty)\right)$.

(1) If $\int_{x}^{\infty} m(y) / n(y) \mathrm{d} y \leq c_{1} m(x)$ and $\int_{x}^{\infty} m(y) \mathrm{d} y \leq c_{2} m(x)$ for all $x \geq x_{0}$. Then for every $\gamma \in\left[0,1 / c_{2}\right)$, we have

$$
\int_{x}^{\infty} e^{\gamma\left(y-x_{0}\right)} \frac{m(y)}{n(y)} \mathrm{d} y \leq \frac{c_{1}}{1-\gamma c_{2}} e^{\gamma\left(x-x_{0}\right)} m(x), \quad x \geq x_{0} .
$$

(2) If $\left(x-x_{0}\right) \int_{x_{0}}^{\infty} m(y) d y \leq c$ for all $x \geq x_{0}$, then for every $\gamma \in[0,1)$, we have

$$
\int_{x}^{\infty}\left(y-x_{0}\right)^{\gamma} m(y) d y \leq \frac{c}{1-\gamma}\left(x-x_{0}\right)^{\gamma-1}, \quad x \geq x_{0} .
$$

Proof. Here, we prove part (1) only since the proof of (2) is simpler. Without loss of generality, assume that $m(x)$ has finite support. Set $M(x)=\int_{x}^{\infty} m(y) / n(y) \mathrm{d} y$. Then

$$
\begin{aligned}
\int_{x}^{\infty} e^{\gamma\left(y-x_{0}\right)} \frac{m(y)}{n(y)} \mathrm{d} y & =-\int_{x}^{\infty} e^{\gamma\left(y-x_{0}\right)} \mathrm{d} M(y) \\
& \leq c_{1} e^{\gamma\left(x-x_{0}\right)} m(x)+c_{1} \gamma \int_{x}^{\infty} e^{\gamma\left(y-x_{0}\right)} m(y) \mathrm{d} y .
\end{aligned}
$$

Consider the special case that $n(x) \equiv 1$ and $c_{1}=c_{2}$. Then, (6.2) gives us

$$
\int_{x}^{\infty} e^{\gamma\left(y-x_{0}\right)} m(y) \mathrm{d} y \leq \frac{c_{2}}{1-\gamma c_{2}} e^{\gamma\left(x-x_{0}\right)} m(x), \quad x \geq x_{0} .
$$

Inserting this into (6.2), we obtain the required assertion.

Proof of Corollary 2.5. a) Note that

$$
C(u)-C(x) \leq \varepsilon \int_{x}^{u} \frac{\mathrm{d} y}{c_{1}+y-x_{0}}=\varepsilon \log \frac{c_{1}+u-x_{0}}{c_{1}+x-x_{0}}, \quad u>x .
$$


We have for $f(x)=\left(c_{1}+x-x_{0}\right)^{\delta}$,

$$
\begin{aligned}
I(f)(x) & \leq \frac{1}{c \delta\left(c_{1}+x-x_{0}\right)^{\delta+\varepsilon-1}} \int_{x}^{\infty} \frac{\left(c_{1}+u-x_{0}\right)^{\delta+\varepsilon}}{\left(c_{1}+u-x_{0}\right)^{\gamma}} \mathrm{d} u \\
& =\frac{-1}{c \delta(\delta+\varepsilon-\gamma+1)} \cdot \frac{1}{\left(c_{1}+x-x_{0}\right)^{\gamma-2}} \\
& \leq \frac{-1}{c \delta(\delta+\varepsilon-\gamma+1)} \cdot \frac{1}{c_{1}^{\gamma-2}} \quad(\gamma \geq 2) .
\end{aligned}
$$

Setting $\delta=(\gamma-\varepsilon-1) / 2$, we prove part (1) of the corollary. Obviously, when $\gamma=2$, $c_{1}$ is allowed to be zero.

b) For part (2), by assumption, we have

$$
\begin{aligned}
C(u)-C(x) & \leq \varepsilon_{1}(u-x)+\varepsilon_{2}\left(u^{2}-x^{2}\right) / 2-\varepsilon_{2}(u-x) x_{0} \\
& =\left[\varepsilon_{1}+\varepsilon_{2}\left(x-x_{0}\right)\right](u-x)+\varepsilon_{2}(u-x)^{2} / 2 .
\end{aligned}
$$

Without loss of generality, assume that $\inf _{x} a(x)=1$. Consider the test function $f(x)=\left(c_{1}-\varepsilon_{2}\left(x-x_{0}\right)\right) e^{\delta\left(x-x_{0}\right)}, \delta>0$. We obtain

$$
\begin{aligned}
I(f) & \leq \frac{1}{-\varepsilon_{2}+c_{1} \delta-\varepsilon_{2} \delta\left(x-x_{0}\right)} \int_{x}^{\infty}\left[c_{1}-\varepsilon_{2}\left(u-x_{0}\right)\right] e^{\left[\varepsilon_{1}+\delta+\varepsilon_{2}\left(x-x_{0}\right)\right](u-x)+\varepsilon_{2}(u-x)^{2} / 2} \\
& =\frac{1}{-\varepsilon_{2}+c_{1} \delta-\varepsilon_{2} \delta\left(x-x_{0}\right)} \int_{0}^{\infty}\left[c_{1}-\varepsilon_{2} u-\varepsilon_{2}\left(x-x_{0}\right)\right] e^{\left[\varepsilon_{1}+\delta+\varepsilon_{2}\left(x-x_{0}\right)\right] u+\varepsilon_{2} u^{2} / 2} \mathrm{~d} u \\
& =\frac{1}{-\varepsilon_{2}+c_{1} \delta-\varepsilon_{2} \delta\left(x-x_{0}\right)}\left[\left(c_{1}+\varepsilon_{1}+\delta\right) \int_{0}^{\infty} e^{\left[\varepsilon_{1}+\delta+\varepsilon_{2}\left(x-x_{0}\right)\right] u+\varepsilon_{2} u^{2} / 2} \mathrm{~d} u+1\right] .
\end{aligned}
$$

If $\varepsilon_{1}<0$, by setting $c_{1}=\delta=-\varepsilon_{1} / 2$, we get

$$
I(f) \leq \frac{1}{-\varepsilon_{2}+\varepsilon_{1}^{2} / 4} .
$$

Next, assume that $\varepsilon_{1} \geq 0$ and set $c_{1}=0$. Since $\varepsilon_{2}<0$, by (6.3), we have

$$
\begin{aligned}
I(f) & \leq \frac{1}{-\varepsilon_{2}-\varepsilon_{2} \delta\left(x-x_{0}\right)}\left[\left(\varepsilon_{1}+\delta\right) \int_{0}^{\infty} e^{\left[\varepsilon_{1}+\delta\right] u+\varepsilon_{2} u^{2} / 2} \mathrm{~d} u+1\right] \\
& \leq \frac{1}{-\varepsilon_{2}}\left[\left(\varepsilon_{1}+\delta\right) \int_{0}^{\infty} e^{\left[\varepsilon_{1}+\delta\right] u+\varepsilon_{2} u^{2} / 2} \mathrm{~d} u+1\right] \\
& =\frac{1}{-\varepsilon_{2}}\left[1+\int_{0}^{\infty} e^{u+\varepsilon_{2} u^{2} /\left[2\left(\varepsilon_{1}+\delta\right)^{2}\right]} \mathrm{d} u\right] .
\end{aligned}
$$

Then by letting $\delta \downarrow 0$, we obtain the estimate in the middle of the expression.

The last estimate in the expression simply follows from (6.3) and Lemma 6.2 (1) with the choice of $m(x)=e^{C(x)}, n(x) \equiv 1, f(x)=e^{\gamma\left(x-x_{0}\right)}$ and

$$
\gamma=\frac{1}{2}\left[\int_{0}^{\infty} e^{\varepsilon_{1}+\varepsilon_{2} u^{2} / 2} \mathrm{~d} u\right]^{-1}
$$

c) To prove part (3), simply apply Lemma 6.1 (1) to $m(x)=e^{C(x)}, n(x)=a(x)$, $f(x)=e^{\gamma\left(x-x_{0}\right)}$ and $\gamma=\frac{1}{2 c_{2}}$.

d) Part (4) also follows from Lemma 6.1 (1) but with $m(x)=e^{C(x)} / a(x), n(x) \equiv$ $1, f(x)=e^{\gamma\left(x-x_{0}\right)}$ and $\gamma=\frac{1}{2 c}$. The particular assertion is then deduced by using the Mean Value Theorem.

e) Finally, part (5) follows from Corollary 6.1 (2) by setting $m(x)=1 / a(x)$, $f(x)=\sqrt{x-x_{0}}$ and $\gamma=1 / 2$. 
Proof of Corollary 2.6. Let $x_{0}=0$ for simplicity. By assumption, there exists $N>$ 0 so that

$$
f^{\prime}(x)>0 \text { for all } x \geq N \text { and } \sup _{x \geq N} I(f)(x)<\infty .
$$

Since $f_{1}^{\prime}(x)=c /(1+x)^{2}+f^{\prime}(x)$, we have $f_{1}^{\prime}(x) \geq f^{\prime}(x)>0$ for all $x \geq N$. As for $x \leq N$, choose $c$ small enough so that $f_{1}^{\prime}(x) \geq c /(1+N)^{2}+\min _{x \leq N} f^{\prime}(x)>0$. We now fix $c$. Because $f$ is an increasing function, there exits $M>0$ such that $f_{1}(x) \leq c+f(x) \leq M f(x)$ for all $x \geq N$. Thus, for $x \geq N$, we have

$$
0<I\left(f_{1}\right)(x) \leq M I(f)(x)<\infty .
$$

Finally, for $x \leq N$, we have

$$
0<I\left(f_{1}\right)(x)=\frac{e^{-C(x)}}{f_{1}^{\prime}(x)} \int_{x}^{N} \frac{f_{1}(u) e^{C(u)}}{a(u)} \mathrm{d} u+\frac{f_{1}^{\prime}(N)}{f_{1}^{\prime}(x)} e^{C(N)-C(x)} I\left(f_{1}\right)(N) .
$$

The right-hand side is bounded in $\left[x_{0}, N\right]$ and so the required conclusion follows from (2.4).

Proof of Corollary 2.7. For a proof of (2.18), refer to [7, Proof a) of Theorem 1.3].

a) Consider the case that $K(r) \leq \inf _{x \geq r}\left[-\left(a^{\prime}+b\right)(x) /\left(x-x_{0}\right)-b^{\prime}(x)\right]+\sup _{y} b^{\prime}(y)$. Fixed $r_{1} \in\left(x_{0}, \infty\right)$ so that $K\left(r_{1}\right)>0$. Otherwise, we have nothing to do. Define

$$
f(x)=\int_{x_{0}}^{x} \mathrm{~d} y \exp \left[-\int_{x_{0}}^{y} \frac{u-x_{0}}{a(u)}\left[K\left(r_{1}\right)-K(u)\right] I_{\left\{u \leq r_{1}\right\}} \mathrm{d} u\right], \quad x>x_{0} .
$$

Since $f^{\prime \prime} \leq 0, f^{\prime}$ is decreasing and so $f(x) \geq\left(x-x_{0}\right) f^{\prime}(x)$. By (2.2), we have

$$
\begin{aligned}
-b^{\prime}(x)-\frac{a f^{\prime \prime}+\left(a^{\prime}+b\right) f^{\prime}}{f}(x) & =\beta_{0}+\frac{-a f^{\prime \prime}-\left(a^{\prime}+b\right) f^{\prime}+\left(-b^{\prime}-\beta_{0}\right) f}{f}(x) \\
& \geq \beta_{0}+\frac{-a f^{\prime \prime}-\left[a^{\prime}+b+\left(x-x_{0}\right)\left(b^{\prime}+\beta_{0}\right)\right] f^{\prime}}{f}(x) \\
& \geq \beta_{0}+K\left(r_{1}\right)\left(x-x_{0}\right) f^{\prime}(x) / f(x) .
\end{aligned}
$$

Here in the last step, we have used the properties of $f$ just mentioned above. Noticing that $\left(x-x_{0}\right) / f(x)$ is non-decreasing, we have $\left(x-x_{0}\right) f^{\prime}(x) / f(x)=$ $\left(x-x_{0}\right) f^{\prime}\left(r_{1}\right) / f(x) \geq\left(r_{1}-x_{0}\right) f^{\prime}\left(r_{1}\right) / f\left(r_{1}\right)$ for all $x \geq r_{1}$. This completes the proof of the main case.

b) The proof of the alternative case is similar, but uses (2.5) instead of (2.2).

To conclude this section, we discuss when the equality in (1.3) holds. Suppose that we have a $C^{2}$-eigenfunction $f$ of $\lambda_{1}>0$. That is, $-a f^{\prime \prime}-b f^{\prime}=\lambda_{1} f$ with $f^{\prime}\left(x_{0}\right)=0$. Then, as we will prove later, $f$ has the following properties: i) $f \in L^{1}(\pi)$, ii) $f^{\prime}>0$ (or $<0$ ) on $\left(x_{0}, \infty\right)$ and iii) $\lim _{x \rightarrow \infty} f^{\prime}(x) e^{C(x)}=-\lambda_{1} \pi(f) Z$. Now, by (6.1) we have $-\left(f^{\prime} e^{C}\right)^{\prime}=\lambda_{1} f e^{C} / a$. Thus

$$
f^{\prime}(x) e^{C(x)}=\lambda_{1} \int_{x}^{\infty} f e^{C} / a-\lambda_{1} \pi(f) Z \geq \lambda_{1} \int_{x}^{\infty}[f-\pi(f)] e^{C} / a
$$

since $\pi(f) \leq 0$ by ii) and iii). Set $\tilde{f}=f-\pi(f)$. We have $\pi(\tilde{f})=0$ and $\tilde{f}^{\prime}(x) e^{C(x)} \geq$ $\lambda_{1} \int_{x}^{\infty} \tilde{f} e^{C} / a$. Hence $I(\tilde{f})(x)^{-1} \geq \lambda_{1}$ for all $x>x_{0}$. Combining this with part $(2)$ of Theorem 2.1, we conclude that the equality of (1.3) holds. 
The remainder of this section is to prove i) - iii) listed above. Where the second one is essential, from which the first one and then the last one follows immediately from the next lemma.

Lemma 6.2. If $L f=-\lambda f$ on $[p, q] \subset[0, \infty)$ for some $\lambda \neq 0$, then we have

$$
-\lambda \int_{p}^{q} f \mathrm{~d} \pi=\left[\left(f^{\prime} e^{C}\right)(q)-\left(f^{\prime} e^{C}\right)(p)\right] / Z \text {. }
$$

Proof. Simply use (6.1).

Lemma 6.3. Let $L f=-\lambda f$ for some $f \in C^{2}\left[x_{0}, \infty\right)$ and for some $\lambda \geq 0$. If there exist $\alpha<\beta$ such that $f \equiv 0$ on $[\alpha, \beta]$, then $f \equiv 0$.

Proof. Due to $a(x)>0$, the assertion is indeed a consequence of the maximum principle (pointed out to the authors by Z. D. Huan). A simple probabilistic proof goes as follows. If $f \not \equiv 0$, without loss of generality, assume that $\gamma:=\inf \{x \geq \beta$ : $f(x) \neq 0\}<\infty$ and there exists $x_{n} \downarrow \gamma$ so that $f\left(x_{n}\right)>0$ (one may replace $f$ with $-f$ if necessary). For each $n \geq 1$, choose $y_{n} \in\left[\gamma, x_{n}\right)$ such that $f\left(y_{n}\right)=\min \{f(x)$ : $\left.x \in\left[\gamma, x_{n}\right]\right\}$. Then $f\left(y_{n}\right) \leq 0$ since $f(\gamma)=0$. Let $x_{t}$ be the $L$-diffusion process starting from $y_{n}$ and set $\tau_{n}=\inf \left\{t \geq 0: x_{t} \in\left\{x_{n}, \gamma-n^{-1}\right\}\right\}$. Then

$$
\mathbb{E} f\left(x_{t \wedge \tau_{n}}\right)=f\left(y_{n}\right)-\lambda \mathbb{E} \int_{0}^{t \wedge \tau_{n}} f\left(x_{s}\right) \mathrm{d} s \leq f\left(y_{n}\right)\left[1-\lambda \mathbb{E} \tau_{n}\right] .
$$

This implies that $\mathbb{E} f\left(x_{\tau_{n}}\right) \leq 0$ for large enough $n$. But for any $n$ with $\gamma-n^{-1} \geq$ $\alpha$, we have $\mathbb{E} f\left(x_{\tau_{n}}\right) \geq f\left(x_{n}\right) \mathbb{P}\left[x_{\tau_{n}}=x_{n}\right]>0$. The contradiction implies the assertion.

Proposition 6.4. Suppose that $\lambda_{1}>0$ and $L f=-\lambda_{1} f$ for some $f \in C^{2}\left[x_{0}, \infty\right)$, $f \neq$ constant and $f^{\prime}\left(x_{0}\right)=0$. Then $f^{\prime} \neq 0$ on $\left(x_{0}, \infty\right)$ and furthermore $f \in L^{1}(\pi)$.

Proof. Suppose that there is a $p>x_{0}$ such that $f^{\prime}(p)=0$.

a) We claim that $f \neq$ constant on $\left[x_{0}, p\right]$. Otherwise, we have $f=-\lambda_{1}^{-1} L f=0$ on $\left[x_{0}, p\right]$ which implies that $f \equiv 0$ by Lemma 6.3 . We now prove that $f(p) \neq 0$. To do so, set $g=f I_{\left[x_{0}, p\right]}+f(p) I_{(p, \infty)}$. If $f(p)=0$, then $g \in C^{2}, L g=-\lambda_{1} g$ and $g \equiv 0$ on $[p, \infty)$. By Lemma 6.3 , we have $g \equiv 0$ and in particular $f \equiv 0$ on $\left[x_{0}, p\right]$. This again implies $f \equiv 0$ on $\left[x_{0}, \infty\right)$ by Lemma 6.3 .

b) By using Lemma 6.2, we have

$$
\int_{x_{0}}^{p} f \mathrm{~d} \pi=0, \quad \int_{x_{0}}^{p} a f^{\prime 2} \mathrm{~d} \pi=-\int_{x_{0}}^{p}(f L f) \mathrm{d} \pi=-\lambda_{1} \int_{x_{0}}^{p} f^{2} \mathrm{~d} \pi .
$$

Here in the last step, we have used the assumption $L f=-\lambda_{1} f$.

c) Without loss of generality, assume that $f(p)=1$. Then $\pi(g)=\pi[p, \infty)<1$. Therefore, by (6.4), we get

$$
\begin{aligned}
\lambda_{1} & \leq \frac{\pi\left(a g^{\prime 2}\right)}{\pi\left(g^{2}\right)-\pi(g)^{2}}=\frac{\pi\left(a g^{\prime 2}\right)}{\int_{x_{0}}^{p} f^{2} \mathrm{~d} \pi+\pi[p, \infty)-\pi[p, \infty)^{2}} \\
& =\frac{\lambda_{1} \int_{x_{0}}^{p} f^{2} \mathrm{~d} \pi}{\int_{x_{0}}^{p} f^{2} \mathrm{~d} \pi+\pi[p, \infty)-\pi[p, \infty)^{2}}<\lambda_{1} .
\end{aligned}
$$

This is a contradiction.

d) Having the increasing property of $f$ in mind, the last assertion of the lemma follows from [2, Lemma 4.13 and Lemma 4.11]. 


\section{Proof of Theorem 3.1 And its Corollaries}

Proof of Theorem 3.1. Here we prove part (2) only since the proof of part (1) is similar and even simpler. Choose $\delta>0$ such that

$$
\int_{x_{0}}^{\infty} \frac{f(x) e^{C(x)}}{a(x)} \mathrm{d} x=\delta \int_{x_{0}}^{-\infty} \frac{f(x) e^{C(x)}}{a(x)} \mathrm{d} x .
$$

Define

$$
g(x)= \begin{cases}\int_{x_{0}}^{x} f^{\prime}(y) I(f)(y) \mathrm{d} y, & x \geq x_{0} \\ \delta \int_{x_{0}}^{x} f^{\prime}(y) I^{-}(f)(y) \mathrm{d} y, & x<x_{0} .\end{cases}
$$

Note that $f\left(x_{0}\right)=0$. We have $g \in C^{2}(\mathbf{R})$ and $g^{\prime}>0$. Next, let $d(x, y)=$ $|g(x)-g(y)|$. Then the proof of Theorem 2.1 gives us

$$
\widetilde{L} d(x, y) \leq \begin{cases}-\delta_{1}^{-1} d(x, y), & \text { if } x>y \geq x_{0} \\ -\delta_{2}^{-1} d(x, y), & \text { if } x_{0} \geq x>y\end{cases}
$$

As for $x>x_{0}>y$, we have

$$
\widetilde{L} d(x, y)=\left[\widetilde{L} g(x)-\widetilde{L} g\left(x_{0}\right)\right]+\left[\widetilde{L} g\left(x_{0}\right)-\widetilde{L} g(y)\right] \leq-\left(\delta_{1} \vee \delta_{2}\right)^{-1} d(x, y) .
$$

The proof is then completed by using Theorem 5.3.

Having Theorem 3.1 in mind, the proofs of Corollaries 3.2-3.5 are parallel to Corollaries 2.4-2.7 respectively and hence omitted.

To conclude this section, we study the same problem as in the last part of Section 7. Note that the comment before Lemma 6.2 is the same.

Proposition 7.1. Suppose that $\lambda_{1}>0$ and $L f=-\lambda_{1} f$ for some $f \in C^{2}(\mathbf{R}) \cap$ $L^{2}(\pi), f \neq$ constant and $(1+|f|) f^{\prime} e^{C}(x) \rightarrow 0$ as $x \rightarrow \infty$. Then $f^{\prime} \neq 0$.

Proof. a) Suppose that there is a $p$ so that $f^{\prime}(p)=0$. Then, we should have $f(p) \neq 0$. Otherwise, set $g=f I_{[p, \infty)}+f(p) I_{(-\infty, p)}$. Then, $f^{\prime \prime}(p)=-\left[\left(b f^{\prime}+\right.\right.$ $\left.\left.\lambda_{1} f\right) / a\right](p)=0$. Hence, $g \in C^{2}(\mathbf{R}), L g=-\lambda_{1} g$ and $g \equiv 0$ on $(-\infty, p]$. By Lemma 6.3 , we have $g \equiv 0$ and hence $f \equiv 0$ which is impossible.

b) Without loss of generality, assume that $f(p)=1=Z$. Note that $f^{\prime} f e^{C}(x) \rightarrow$ 0 as $x \rightarrow \infty$, we have

$$
\begin{aligned}
\int_{p}^{\infty} a f^{\prime 2} \mathrm{~d} \pi & =\int_{p}^{\infty} f^{\prime 2} e^{C}(x) \mathrm{d} x=-\int_{p}^{\infty} f\left(f^{\prime} e^{C}\right)^{\prime} \mathrm{d} x \\
& =-\int_{p}^{\infty}(f L f) \mathrm{d} \pi=-\lambda_{1} \int_{p}^{\infty} f^{2} \mathrm{~d} \pi .
\end{aligned}
$$

On the other hand, it follows from Lemma 6.2 that $\int_{p}^{\infty} f \mathrm{~d} \pi=0$ and so $\pi(g)=$ $\pi(-\infty, p)<1$. Hence

$$
\lambda_{1} \leq \frac{\pi\left(a g^{\prime 2}\right)}{\pi\left(g^{2}\right)-\pi(g)^{2}}=\frac{\int_{p}^{\infty} a f^{2} \mathrm{~d} \pi}{\int_{p}^{\infty} f^{2} \mathrm{~d} \pi+\pi[-\infty, p)-\pi[-\infty, p)^{2}}<\lambda_{1} .
$$

This is a contradiction. 


\section{Proofs of Theorem 4.1, Theorem 4.6 and Their Corollaries}

Proof of Theorem 4.1. Part (1) follows directly from (4.1)-(4.4). The conclusion (4.7) follows by replacing the function $f$ in (4.4) with

$$
g(r)=\int_{0}^{r} e^{-C(s)} d s \int_{s}^{D} \frac{f(u) e^{C(u)}}{\alpha(u)} \mathrm{d} u .
$$

Then (4.8) follows from (4.7) by using the Mean Value Theorem.

Proof of Corollary 4.2. We remark that the corollary in the present case is deduced directly from part (1) of Theorem 4.1 by taking

$$
f(r)=\int_{0}^{r} \mathrm{~d} s \exp \left[-\int_{0}^{s} \frac{u}{\alpha(u)}\left[K\left(r_{1}\right)-K(u)\right] I_{\left\{u \leq r_{1}\right\}} \mathrm{d} u\right], \quad r \in[0, D) .
$$

The details are very much the same as in the proof of Corollary 2.7.

Proof of Corollary 4.4. Simply use Lemma 5.1.

Proof of Theorem 4.6. By Proposition 4.5, we may assume that $a=\operatorname{diag}\left\{a_{1}, \cdots, a_{d}\right\}$ and then $\bar{b}_{i}=b_{i}, i \leq d$.

a) When $j=1$, we may assume that $\int_{0}^{1} s\left|K_{1}(s)\right| \mathrm{d} s<\infty$. Let $\widetilde{L}$ be the coupling operator with $[14]$

$$
c(x, y)=\sqrt{a(x)}\left(\sqrt{a(y)}-2 \frac{\sqrt{a(y)^{-1}}(x-y)(x-y)^{*}}{\left|\sqrt{a(y)^{-1}}(x-y)\right|^{2}}\right) .
$$

Take $d(x, y)=|x-y|$ and choose $\alpha(r)=\alpha_{1}(r)$ and $K(r)=K_{1}(r)$ (see [18]). Let $r_{1} \in(0, D)$ so that $K_{1}\left(r_{1}\right)>0$ and define $f(r)$ as in (8.1) but replacing $K$ with $K_{1}$. It follows from Corollary 4.2 that $\lambda^{*} \geq K\left(r_{1}\right) \inf _{s \in\left(0, r_{1}\right)} f^{\prime}(s) / f(s)$. Next, for $n \geq 1$, let $D_{n}=\prod_{i=1}^{d}[-n, n]$. Since $a=\operatorname{diag}\left\{a_{1}, \cdots, a_{d}\right\}$, the normal vector on $\partial S_{n}$ coincides with that under the Riemannian metric $\left(g\left(\partial_{i}, \partial_{j}\right)\right)=a^{-1}$. Then $d(x, y):=f(|x-y|)$ satisfies the boundary condition given in Theorem 5.3. From this we claim that $\operatorname{gap}(L) \geq \lambda^{*}$ and so the assertion of the theorem in the case of $j=1$ follows.

b) Take $d(x, y)=\rho(x, y)$ and $c(x, y)=\sqrt{a(x)}\left(I-2 u u^{*}\right) \sqrt{a(y)}$, where $u_{i}=$ $\frac{1}{\rho(x, y)} \int_{x_{i}}^{y_{i}} \frac{1}{\sqrt{a_{i}(r)}} \mathrm{d} r, x \neq y$. Then the proof for the case of $j=2$ is similar to that for $j=1$ (refer to [6, Theorem 4.2]).

c) To prove the case of $j=3$, we use the coupling by reflection and take $d(x, y)=$ $|x-y|_{1}$. For $x_{0} \geq y_{0}$, Lemma 5.2 gives $d\left(x_{t}, y_{t}\right)=\sum_{i=1}^{d}\left(x_{i}(t)-y_{i}(t)\right), P^{x_{0}, y_{0} \text {-a.s. }}$ On the other hand, for $d(x, y)=\sum_{i}\left(x_{i}-y_{i}\right)$, we have

$$
A(x, y) \geq \alpha_{2}(r), \quad K_{3}(r) \leq-\sup _{x \geq y, d(x, y) \geq r} B(x, y) .
$$

Next, let $u_{n}$ be the first Neumann eigenfunction on $D_{n}$, then there exists $x \geq y$ such that $u_{n}(x) \neq u_{n}(y)$. Lemma 2.1 and the proof of [15, Lemma 2.4] then give $\operatorname{gap}\left(D_{n}\right) \geq \delta$. This proves the theorem in the case of $j=3$.

Proof of Corollary 4.7. We consider the cases of $j=1$ and $j=3$ only since the proof of $j=2$ is similar. Actually, by [7, Theorem 1.3], the lower bound given for $j=2$ is also a lower bound of the logarithmic Sobolev constant. To see this, take the Riemannian metric $g\left(\partial_{i}, \partial_{j}\right)=\delta_{i j} a_{i}^{-1}$. Then $\left\{X_{i}\right\}$ is a normal orthogonal basis 
with $\nabla_{X_{i}} X_{j}=0$ for all $i, j$, the sectional curvature is zero, $\rho$ is the Riemannian distance and $L=\Delta_{g}+\nabla_{g} \bar{V}$.

a) For $|x-y|=r$, let $\psi(s)=x+s(y-x), s \in[0,1]$. We have

$$
\begin{aligned}
\| \sigma(x) & -\sigma(y) \|^{2}-|x-y|^{-2}|(\sigma(x)-\sigma(y))(x-y)|^{2}+\langle\bar{b}(x)-\bar{b}(y), x-y\rangle \\
& \leq r^{2} \kappa\left(1-d^{-1}\right)+\sum_{i, j=1}^{d}\left(x_{i}-y_{i}\right)\left(x_{j}-y_{j}\right) \int_{0}^{1} \partial_{i} \bar{b}_{i}(\psi(u)) \mathrm{d} u \\
& \leq r^{2} \kappa\left(1-d^{-1}\right)-r^{2} \lambda_{\min }\left(-\int_{0}^{1} \partial_{j} \bar{b}_{i}(\psi(u)) \mathrm{d} u\right) \\
& \leq r^{2} \kappa\left(1-d^{-1}\right)-r^{2} \int_{0}^{1} \lambda_{\min }\left(-\partial_{j} \bar{b}_{i}(\psi(u)) \mathrm{d} u .\right.
\end{aligned}
$$

Next, choose $u_{0} \in[0,1]$ such that $\left|\psi\left(u_{0}\right)-p\right|=\min _{u \in[0,1]}|\psi(u)-p|$. Then $\mid \psi(u)-$ $p|\geq| \psi(u)-\psi\left(u_{0}\right)|=| u-u_{0} \mid r$. Note that $\theta_{1}$ is non-decreasing, we obtain

$$
\int_{0}^{1} \lambda_{\min }\left(-\partial_{j} \bar{b}_{i}(\psi(u))\right) \mathrm{d} u \geq \int_{0}^{1} \theta_{1}\left(\left|u-u_{0}\right| r\right) \mathrm{d} u \geq \frac{2}{r} \int_{0}^{r / 2} \theta_{1}(u) \mathrm{d} u=\gamma_{1}(r / 2) .
$$

Hence we can take $K_{3}(r)=\gamma_{3}(r / 2)$.

b) Finally, note that

$$
\begin{aligned}
\sum_{i=1}^{d}\left(\bar{b}_{i}(y)-\bar{b}_{i}(x)\right) & =\sum_{j=1}^{d}\left(y_{j}-x_{j}\right) \int_{0}^{1} \sum_{i=1}^{d} \partial_{j} \bar{b}_{i}(\psi(u)) \mathrm{d} u \\
& \leq-|y-x|_{1} \int_{0}^{1}\left(-\max _{j} \sum_{i=1}^{d} \partial_{j} \bar{b}_{i}(\psi(u))\right) \mathrm{d} u \\
& \leq-|y-x|_{1} \int_{0}^{1} \theta_{3}(|\psi(u)-p|) \mathrm{d} u \leq-|y-x|_{1} \gamma_{3}(r / 2),
\end{aligned}
$$

we can take $K_{3}(r)=\gamma_{3}(r / 2)$.

\section{Proof of Theorem 4.13}

Lemma 9.1. Let $\left(x_{t}, y_{t}\right)$ be a coupling of the L-diffusion process. If

$$
E^{x, y}\left|x_{t}-y_{t}\right|^{2} \leq|x-y|^{2} \exp [2 c t]
$$

for all $t \geq 0, x, y \in \mathbf{R}^{d}$ and some $c \in \mathbf{R}$, then we have $\left|\nabla P_{t} f\right|^{2} \leq \exp [2 c t] P_{t}|\nabla f|^{2}$ for all $t \geq 0$ and $f \in C_{0}^{1}\left(\mathbf{R}^{d}\right)$.

Proof. Since $f \in C_{0}^{1}\left(\mathbf{R}^{d}\right)$, for any $\varepsilon>0$, there exists $\delta>0$ such that

$$
\frac{|f(x)-f(y)|}{|x-y|} \leq|\nabla f(x)|+\varepsilon, \quad|x-y| \in(0, \delta) .
$$


Let $T$ be the coupling time. We have

$$
\begin{aligned}
\frac{\left|P_{t} f(x)-P_{t} f(y)\right|}{|x-y|} & \leq E^{x, y}\left\{\frac{\left|f\left(x_{t}\right)-f\left(y_{t}\right)\right|}{\left|x_{t}-y_{t}\right|} \cdot \frac{\left|x_{t}-y_{t}\right|}{|x-y|} I_{\{T>t\}}\right\} \\
& \leq \exp [c t]\left\{E^{x, y} \frac{\left|f\left(x_{t}\right)-f\left(y_{t}\right)\right|^{2}}{\left|x_{t}-y_{t}\right|^{2}} I_{\{T>t\}}\right\}^{1 / 2} \\
& \leq \exp [c t]\left\{E^{x, y}\left(\left|\nabla f\left(x_{t}\right)\right|+\varepsilon\right)^{2}+\|\nabla f\|_{\infty} P^{x, y}\left(\left|x_{t}-y_{t}\right| \geq \delta\right)\right\}^{1 / 2} \\
& \leq \exp [c t]\left\{P_{t}|\nabla f|^{2}(x)+2 \varepsilon\|\nabla f\|_{\infty}\right. \\
& \left.+\varepsilon^{2}+\|\nabla f\|_{\infty} \delta^{-2}|x-y|^{2} \exp [2 c t]\right\}^{1 / 2}
\end{aligned}
$$

The assertion now follows by letting $y \rightarrow x$ and then $\varepsilon \rightarrow 0$.

Proof of Theorem 4.13. a) Suppose that $f \in C_{0}^{1}\left(\mathbf{R}^{d}\right)$. Let $\widetilde{L}$ be the operator of march coupling (see [3] or [4]), i.e., $c(x, y)=\sigma(x) \sigma(y)^{*}$. Let $h(x, y)=|x-y|^{2}$, we have

$$
\widetilde{L} h(x, y)=2\|\sigma(x)-\sigma(y)\|^{2}+2\langle b(x)-b(y), x-y\rangle \leq 2 K h(x, y), \quad x, y \in \mathbf{R}^{d} .
$$

Then $E^{x, y}\left|x_{t}-y_{t}\right|^{2} \leq|x-y|^{2} \exp [2 K t], t \geq 0$. By Lemma 9.1 we have

$$
\left|\nabla P_{t} f\right|^{2} \leq \exp [2 K t] P_{t}|\nabla f|^{2}, \quad f \in C_{0}^{1}\left(\mathbf{R}^{d}\right) .
$$

For given $t>0$, let $H(r)=P_{r}\left(P_{t-r} f\right)^{2}, r \in[0, t]$. By (9.1) we have

$$
\begin{aligned}
H^{\prime}(r) & =P_{r} L\left(P_{t-r} f\right)^{2}-2 P_{r}\left(P_{t-r} f\right) L P_{t-r} f \\
& =2 P_{r}\left\langle a \nabla P_{t-r} f, \nabla P_{t-r} f\right\rangle \leq 2 \bar{a} \exp [2 K(t-r)] P_{t}|\nabla f|^{2} .
\end{aligned}
$$

By integrating over $r$ from 0 to $t$, we obtain (4.14).

b) In general, fix $x$ and $t$, let $\pi_{t}=\delta_{x} P_{t}$. Next, given $f \in C^{1}\left(\mathbf{R}^{d}\right)$ with $\pi_{t}(f)=0$ and $\pi_{t}\left(f^{2}\right)=1$. Let $B_{n}=\{y:|y-x| \leq n\}, n \geq 1$. For any $\varepsilon>0$, there exists $n_{\varepsilon}$ such that $\bar{a} \int_{B_{n}^{c}}\left(|\nabla f|^{2}+f^{2}\right) \mathrm{d} \pi_{t}+\pi_{t}\left(B_{n}^{c}\right)<\varepsilon$ for all $n \geq n_{\varepsilon}$. Choose $h \in C^{\infty}(\mathbf{R})$ such that $0 \leq h \leq 1, h(r)=1$ for $r \leq 0$ and $h(r)=0$ for $r \geq 1$. Let $f_{n}(y)=f(y) h(|y-x|-n)$. Then $f_{n} \in C_{0}^{1}\left(\mathbf{R}^{d}\right)$ and

$$
\begin{gathered}
\bar{a} \int\left|\nabla f_{n}\right|^{2} \mathrm{~d} \pi_{t} \geq \bar{a} \int|\nabla f|^{2} \mathrm{~d} \pi_{t}-\left(\bar{a}\|h\|_{\infty}^{2}+1\right) \varepsilon, \\
\int\left(f_{n}-\int f_{n} \mathrm{~d} \pi_{t}\right)^{2} \mathrm{~d} \pi_{t} \geq 1-3 \varepsilon, \quad n \geq n_{\varepsilon} .
\end{gathered}
$$

Combining these with a) and letting $\varepsilon \rightarrow 0$, we complete the proof.

\section{ACKNOWLEDGEMENTS}

The authors are greatly indebted to Professor R. Durrett for encouragement and to a referee for careful comments on the first version of the paper. 


\section{REFERENCES}

[1] Chavel, I., Eigenvalues in Riemannian Geometry, New York: Academic Press, 1984. MR 80g:58104

[2] Chen, M. F., From Markov Chains to Non-Equilibrium Particle Systems, Singapore: World Scientific, 1992. MR 94a:60135

[3] Chen, M. F., Optimal Markovian couplings and applications, Acta Math. Sin. New Ser. 10:3 (1994), 260-275.

[4] Chen, M. F. and Li, S. F., Coupling methods for multidimensional diffusion processes, Ann. Probab. 17:1(1989), 151-177. MR 90a:60134

[5] Chen, M. F. and Wang, F. Y., Application of coupling method to the first eigenvalue on manifold, Sci. Sin. (A), 37:1(1994), 1-14. MR 96d:58141

[6] Chen, M. F. and Wang, F. Y., Estimation of the first eigenvalue of the second order elliptic operators, J. Funct. Anal. 131:2 (1995), 345-363. MR 96g:35142

[7] Chen, M. F. and Wang, F. Y., Estimates of logarithmic Sobolev constant-An improvement of Bakry-Emery criterion, preprint (1994).

[8] Chen, M. F. and Wang, F. Y., On order-preservation and positive correlations for multidimensional diffusion processes, Probab. Theory Relat. Fields, 95 (1993), 421-428. MR 94g:60147

[9] Fukushima, M., Oshima, Y. and Takeda, M., Dirichlet Forms and Symmetric Markov Processes, Walter de Gruyter \& Co., 1994. MR 96f:60126

[10] Hsu, E. P., Logarithmic Sobolev inequalities on path spaces, C. R. Acad. Sci. Paris Sér I. Math. 320 (1995), 1009-1012. MR 96e:58167

[11] Kac, I. S. and Krein, M. G., Criteria for discreteness of the spectrum of a singular string, Izv. Vyss. Učebn. Zaved. Mat 2 (1958), 136-153 (In Russian). MR 25:3232

[12] Kotani, S. and Watanabe, S., Krein's spectral theory of strings and generalized diffusion processes, Lecture Notes in Math., 923 (1982), 235-259. MR 83h:60081

[13] Liggett, T. M., Exponential $L_{2}$ convergence of attractive reversible nearest systems, Ann. Prob. 17(1989), 403-432. MR 90h:60099

[14] Lindvall, T. and Rogers, L. C. G., Coupling of multidimensional diffusions by reflection, Ann. Probab. 14 (1986), 860-872. MR 88b:60179

[15] Wang, F. Y., Application of coupling method to the Neumann eigenvalue problem, Prob. Theory Relat. Fields, 98 (1994), 299-306. MR 96d:58141

[16] Wang, F. Y., Spectral gap for diffusion processes on non-compact manifolds, Chinese Sci. Bull., 40:14 (1995), 1145-1149. CMP 96:06

[17] Wang, F. Y., Logarithmic Sobolev inequalities for diffusion processes with application to path space, preprint (1995).

[18] Wang, F. Y., Gradient estimates on $\mathbf{R}^{d}$, Canad. Math. Bull., 37:4(1994), 560-570. MR 95i:35042

Department of Mathematics, Beijing Normal University, Beijing 100875, P.R. China

E-mail address: mf chen@ns.bnu.edu.cn 